\title{
The dynamic response of clamped rectangular Y-frame and corrugated core sandwich plates
}

\author{
V. Rubino, V. S. Deshpande ${ }^{1}$ and N.A. Fleck \\ Cambridge University, Engineering Department, Trumpington Street, Cambridge, \\ CB2 1PZ, U.K.
}

\begin{abstract}
The dynamic response of fully clamped, monolithic and sandwich plates of equal areal mass has been measured by loading rectangular plates over a central patch with metal foam projectiles. All plates are made from AISI 304 stainless steel, and the sandwich topologies comprise two identical face-sheets and either Y-frame or corrugated cores. The resistance to shock loading is quantified by the permanent transverse deflection at mid-span of the plates as a function of projectile momentum. At low levels of projectile momentum both types of sandwich plate deflect less than monolithic plates of equal areal mass. However, at higher levels of projectile momentum, the sandwich plates tear while the monolithic plates remain intact. Three-dimensional finite element (FE) calculations adequately predict the measured responses, prior to the onset of tearing. These calculations reveal that the accumulated plastic strains in the front face of the sandwich plates exceed those in the monolithic plates. These high plastic strains lead to failure of the front face sheets of the sandwich plates at lower values of projectile momentum than for the equivalent monolithic plates.
\end{abstract}

Keywords: Sandwich plates, dynamic response, FE simulations, lattice materials.

\footnotetext{
1 Author to whom all correspondence should be addressed. Fax: +44 1223 332662; email: vsd@eng.cam.ac.uk.
} 
Clamped plates are representative of the structures used in the design of commercial and military vehicles. For example, the hull of a ship comprises plates welded to an array of stiffeners. Over the past decade there have been substantial changes in ship design, see for example the review by Paik et al. (2003). One such innovation has been the Schelde Shipbuilding ${ }^{2}$ sandwich design of ship hulls with a Y-frame core, see Fig. 1a. Full-scale ship collision trials reveal that the Y-frame design is more resistant to tearing than conventional monolithic designs, see Wevers and Vredeveldt (1999) and Ludolphy (2001). Likewise, the finite element simulations by Konter et al. (2004) suggest that the Y-frame confers improved perforation resistance. Naar et al. (2002) have argued in broad terms that the ability of the bending-governed Yframe topology to spread the impact load over a wide area, combined with the high inplane stretching resistance of the $\mathrm{Y}$-frame, gives the enhanced performance of the $\mathrm{Y}$ frame sandwich construction over conventional single and double hull designs. The corrugated sandwich core (Fig. 1b) is a competing prismatic topology to that of the Yframe.

Pedersen et al. (2006) and Rubino et al. (2007b) have investigated the quasi-static structural response of Y-frame sandwich beams while Côté et al. (2006) studied the response of sandwich beams with a corrugated core. These studies reveal that geometrical imperfections and/or non-uniform loading (such as indentation loading) induce bending within the struts of the corrugated core and reduce the compressive strength of the corrugated core to approximately that of the Y-frame core. Tilbrook et al. (2007) have performed a combined experimental and numerical investigation into the dynamic compressive response of these cores. They found that buckling of the webs is delayed at low impact velocities by inertial stabilization of the webs while plastic shock wave effects dominate the response at higher impact velocities.

Radford et al. (2005) have developed an experimental technique to subject structures to high intensity pressure pulses using metal foam projectiles. The applied pressure versus time pulse mimics shock loading in air and in water, with peak pressures on the order of $100 \mathrm{MPa}$ and loading times of approximately $100 \mu \mathrm{s}$. This laboratory method has been employed in several investigations on the dynamic response of sandwich beams and plates, see for example Radford et al. (2006a) and Rathbun et al. (2006). Rubino et al. (2007a) have recently used this technique to demonstrate that sandwich beams with Y-frame and corrugated cores have a superior dynamic performance to monolithic beams of equal areal mass; in these tests, the prismatic axis of the cores was aligned with the longitudinal axis of the beams. However, clamped plates are more representative of marine structural components. The main aim of the current study is to determine the relative dynamic performance of clamped

\footnotetext{
${ }^{2}$ Royal Schelde, P.O. Box 164380 AA Vlissingen, The Netherlands.
} 
monolithic plates and clamped sandwich plates with Y-frame and corrugated cores. In order to enable a fair comparison with the beam results in Rubino et al. (2007a) we shall employ the same methodology to load the sandwich plates investigated here.

The outline of the paper is as follows. First, the manufacturing route of the sandwich plates is detailed and the experimental protocol is described for dynamic loading the plates over a central patch by metal foam projectiles. The experimental results are then compared with the corresponding observations for beams with two orientations of the core and also for monolithic plates of equal areal mass. Finally, finite element predictions are used to estimate the onset of failure in the plates.

\section{2.}

\section{Experimental investigation}

Clamped rectangular sandwich plates, made from AISI 304 stainless steel and comprising two identical face-sheets and either a Y-frame or corrugated core, were loaded dynamically with metal foam projectiles as sketched in Fig. 2a. The main aims of this experimental study were:

(i) To measure the shock resistance of rectangular sandwich plates with Y-frame and corrugated cores, and of monolithic plates. All plates have an equal areal mass.

(ii) To compare the dynamic performance of clamped rectangular sandwich plates and of end-clamped beams.

(iii) To investigate the fidelity of three-dimensional finite element calculations in predicting the observed dynamic responses.

\subsection{Specimen configuration and manufacture}

The core and face sheets of the sandwich plates were made from AISI type 304 stainless steel of thickness $t=0.3 \mathrm{~mm}$ and of density $\rho_{f}=7900 \mathrm{kgm}^{-3}$. The Yframe and corrugated cores had an effective density $\rho_{c} \approx 200 \mathrm{kgm}^{-3}$ (corresponding to a relative density $\bar{\rho}=2.5 \%$ ) and a core depth $c=22 \mathrm{~mm}$. All sandwich plates had an areal mass $m=2 t \rho_{f}+c \rho_{c} \approx 10 \mathrm{kgm}^{-2}$.

A co-ordinate system $\left(X_{1}, X_{2}, X_{3}\right)$ is introduced in Fig. 2a in order to clarify the orientations of the cores in the sandwich plates. The longitudinal and transverse axes of the Y-frame and corrugated core are aligned with the $X_{1}$ and $X_{2}$ directions, respectively, while the through thickness direction of the core is along the $X_{3}$ axis. The short edge of the plate between the clamped edges is labelled $b$ along the $X_{2}$ direction while the long edge is labelled $2 L$ along the $X_{1}$ direction; the chosen aspect ratio $b / 2 L=0.52$ matches that of Royal Schelde's hull panels. The total depth of the sandwich plates is denoted $c+2 t$. The cross-sectional dimensions of the Y- 
frame and corrugated core are given in Figs. $2 b$ and $2 c$, respectively. Note that the $Y$ frames and corrugated cores are close-packed along the $X_{2}$-direction such that adjacent cores touch as sketched in Fig. 2.

The manufacturing sequence for the Y-frame sandwich plates is outlined in Fig. 3. The Y-frame webs were computer-numerical-control (CNC) folded to produce a continuous corrugated profile with flat tops. Slots were then CNC-cut along the tops of the corrugations as seen in Fig. 3a. Each corrugated sheet contained five Y-frame webs. The legs of the Y-frame were CNC folded and then teeth were CNC-cut into the bottom of these legs to mate with the slots of the corrugations (Fig. 3a). The assembly was then laser welded together (Fig. 3b). This core assembly was of length $375 \mathrm{~mm}$ and of width $130 \mathrm{~mm}$. Next, the Y-frame was spot welded to $375 \mathrm{~mm} \times 250 \mathrm{~mm}$ stainless steel face sheets (Figs. $3 \mathrm{c}$ and d). The face sheets were then continuous laser welded to the core using $\mathrm{CNC}$ laser welding. In order to ensure intimate contact between the face sheet and the core during the laser welding, an aluminium insert temporarily supported the core (Fig. 3d).

The corrugated core, comprising ten struts (five corrugations) inclined at $\pm 60^{\circ}$ (Fig 2c), was also manufactured by $\mathrm{CNC}$ folding. The core was then laser welded to two identical face sheets using the same procedure as that described above for the Yframe sandwich plates.

In order to minimize rotations and deflections of the sandwich plates along the clamped periphery, the clamping procedure as sketched in Fig. 4 was employed. Along the sides of the sandwich panel, two steel inserts of dimension $375 \mathrm{~mm} \times 60 \mathrm{~mm} \times 22 \mathrm{~mm}$ were glued between the face sheets. The ends of the sandwich core were then dipped in epoxy resin to a depth of $62.5 \mathrm{~mm}$, as sketched in Fig. 4a. The periphery of the sandwich plates was then clamped between two steel frames using M8 bolts, as shown in Fig. 4b. The inner dimensions of the clamping frame were $250 \mathrm{~mm} \times 130 \mathrm{~mm}$ and thus the effective dimensions of the $\mathrm{Y}$-frame and corrugated core sandwich plates (i.e. spans of the plate between the clamped edges) were $2 L=250 \mathrm{~mm}$ and $b=130 \mathrm{~mm}$.

Monolithic rectangular plates of thickness $h=1.2 \mathrm{~mm}$ were also made from the AISI 304 stainless steel; they possessed the same areal mass as the sandwich plates, $m \approx 10 \mathrm{kgm}^{-2}$, and were of overall dimension $375 \mathrm{~mm} \times 250 \mathrm{~mm}$. As for the sandwich case, the monolithic plates were clamped between the steel frame of Fig. $4 \mathrm{~b}$, giving an effective span between the clamped edges of $2 L=250 \mathrm{~mm}$ and $b=130 \mathrm{~mm}$. 


\subsection{Uniaxial response of the constituent materials}

Tensile tests were performed on dog-bone specimens machined from the as-received AISI 304 stainless steel material. The true stress versus logarithmic strain curve $\sigma_{0}\left(\varepsilon^{p}\right)$ of the AISI 304 stainless steel measured at an applied nominal strain-rate of $10^{-3} \mathrm{~s}^{-1}$ is reported in Fig. 5a. The response is linear elastic with a Young's modulus $E=210 \mathrm{GPa}$, followed by linear hardening above a yield strength of $\sigma_{Y}=210 \mathrm{MPa}$. The finite element model requires a description of the high strain rate response of the 304 stainless steel. The strain-rate sensitivity of the AISI 304 stainless steel was documented by Stout and Follansbee (1986) in the range $10^{-4} \mathrm{~s}^{-1}<\&<10^{4} \mathrm{~s}^{-1}$. Following their approach, we define a dynamic strength enhancement ratio $R$ as the ratio of the strength $\sigma_{d}$ (at a plastic strain $\varepsilon^{p}=0.1$ ) at an applied strain-rate $\& \mathbb{Q}$ to the strength $\sigma_{0}\left(\varepsilon^{p}=0.1\right)$ at an applied $\&=10^{-3} \mathrm{~s}^{-1}$. Stout and Follansbee (1986) found that the ratio $R$ is reasonably independent on the choice of plastic strain $\varepsilon^{p}$ at which $R$ is calculated. We make use their measured values for $R(\mathscr{\&})$ and write the dynamic strength $\sigma_{d}$ versus plastic strain $\varepsilon^{p}$ response in the decoupled form:

$$
\sigma_{d}=R(\mathscr{\&}) \sigma_{0}\left(\varepsilon^{p}\right)
$$

This prescription for the strain rate sensitivity of the stainless steel is used in the finite element calculations described in Section $4 ; \sigma_{0}\left(\varepsilon^{p}\right)$ is the measured quasi-static stress versus strain history (Fig. 5a). For illustration, the estimated true tensile stress versus logarithmic plastic strain behaviour of the AISI 304 stainless steel is sketched in Fig 5a at four selected values of applied strain-rate using the $R$ values given by Stout and Follansbee (1986).

The projectiles used to impact the plates are made from Alporas aluminium alloy foam of relative density $\bar{\rho} \approx 0.11$. The compressive response of the foam is required in order to calibrate the constitutive parameters of the finite element model. The quasi-static compressive response of the foam was measured at a strain-rate of $10^{-3} \mathrm{~s}^{-}$ 1 using a cylindrical specimen similar to that of the projectile in the dynamic experiments (diameter $50.8 \mathrm{~mm}$ and length $51 \mathrm{~mm}$ ). The nominal compressive response is shown in Fig. 5: the foam has a plateau strength of approximately $2.5 \mathrm{MPa}$ and a nominal densification strain of $\varepsilon_{D} \approx 0.8$.

\subsection{Protocol for the dynamic tests}

The clamped rectangular monolithic and sandwich plates were impacted by Alporas aluminium foam projectiles over a central circular patch of diameter $d$, as shown in Fig. 2a. Radford et al. (2005) have recently demonstrated that foam projectiles can be used to provide well-characterised pressure versus time loading pulses. This method 
has been used to measure the dynamic response of sandwich beams with lattice cores (Radford et al. 2006a) and circular sandwich plates with metal foam cores (Radford et al. 2006b) and lattice cores (McShane et al. 2006). The impact site of the Y-frame and corrugated core sandwich plates is marked in Fig. 2: in keeping with the Y-frame structures used in ships constructed by Schelde Shipbuilding, the web of the Y-frame was adjacent to the impacted face of the plate while the Y-frame leg was attached to the non-impacted face of the sandwich plate.

Projectiles of length $l_{0} \approx 51 \mathrm{~mm}$ and diameter $d=50.8 \mathrm{~mm}$ were electro-discharge machined into a circular cylindrical shape from Alporas foam blocks of density $\rho_{p}=313 \mathrm{kgm}^{-3}$. The projectiles were fired at a velocity $v_{0}$ in the range $94 \mathrm{~ms}^{-1}$ to $451 \mathrm{~ms}^{-1}$ using a gas gun with circular barrel of diameter $50.8 \mathrm{~mm}$ and length $4.5 \mathrm{~m}$. Results are given in terms of the projectile momentum per unit area $I_{0}=\rho_{p} l_{0} v_{0}$, as listed in Table 1. The plates were inspected after each experiment in order to measure the permanent central deflection and to detect any visible signs of failure.

3.

Experimental results

At least four levels of momentum $I_{0}$ were imparted to the monolithic and sandwich plates by varying the metal foam projectile impact velocity. A summary of the measured deflections, core compression and observed failure modes is provided in Table 1.

\subsection{Effect of projectile momentum upon the deformation of monolithic and sandwich plates}

The dynamic response of the sandwich and monolithic plates is summarised in a plot of permanent back face deflections $w_{b}$ at the centre of the plates versus the initial momentum of the foam projectile $I_{0}$, see Fig. 6a. Both types of sandwich plate have a similar performance over the projectile momentum range $2 \mathrm{kNsm}^{-2} \leq I_{0} \leq 5 \mathrm{kNsm}^{-2}$ and are perforated by the projectile at higher values of $I_{0}$. In contrast, the monolithic plates have higher deflections $w_{b}$ than the sandwich plates but remain intact over the entire range of $I_{0}$ investigated.

Define the permanent core compressive strain as $\varepsilon_{C} \equiv \Delta c / c$, where $\Delta c$ is the reduction in core thickness at the centre of the plate due to impact. The measured dependence of $\varepsilon_{C}$ upon $I_{0}$ is plotted in Fig. $6 \mathrm{~b}$ for the two sandwich plate configurations. Both sandwich plates undergo similar levels of core compression over the range of $I_{0}$ values investigated.

Photographs showing the impacted face of the as-tested plates $\left(I_{0} \approx 5 \mathrm{kNsm}^{-2}\right)$ within the clamping frame are shown in Fig. 7. Tears running parallel to the $X_{1}$-axis are 
observed on the impacted face sheets of both the Y-frame and corrugated core sandwich plates. In contrast, the monolithic plate remained intact at this value of $I_{0}$. Partial pull-out of the monolithic plate from the clamped supports is evident in Fig. 7a: a pen-line was traced along the inner clamped boundaries of the plates prior to each test, and the line moved inwards during the test.

In order to gain insight into the dynamic deformation mechanisms, the monolithic and sandwich plates tested at $I_{0} \approx 3 \mathrm{kNs} \mathrm{m}^{-2}$ were sectioned along their mid-span $X_{1}=0$. Photographs of the sectioned specimens are shown in Fig. 8a for the monolithic plate, in Fig. 8b for the sandwich plate with the Y-frame core and in Fig. 8c for the sandwich plate with the corrugated cores. The sectioned profiles show that significant plastic deformation occurs in the vicinity of the foam impact site and that the plates are continuously curved. It is deduced that the dynamic deformation of plates involves the formation of travelling hinges, similar to the observed behaviour of monolithic and sandwich beams and plates reported by numerous investigators, for example Radford et al. (2006b); McShane et al. (2006) and Rubino et al. (2007a). For both types of sandwich plate, the core has sheared and compressed.

3.2 Comparison of the performance of rectangular sandwich plates and beams Rubino et al. (2007a) investigated the response of clamped Y-frame and corrugated core sandwich beams impacted by metal foam projectiles. Beams with both transverse and longitudinal core orientations were investigated in that study and constituent materials used to manufacture the sandwich beams, the core and face sheet thicknesses and core relative density were identical to those employed in the present investigation. Moreover, the spans of the beams in Rubino et al. (2007a) were $250 \mathrm{~mm}$, that is equal to the length of the longer side of the rectangular plates studied here. Given these similarities between the two studies it is instructive to compare the responses of the Y-frame and corrugated core sandwich beams and plates. In qualitative terms, the deformed shape on the $X_{1}=0$ plane of the plate specimens (Fig. 8a) is in good agreement with that observed by Rubino et al. (2007a) for corrugated and Y-frame sandwich beams in the transverse configuration.

The permanent back face deflections $w_{b}$ at mid-spans of the Y-frame and corrugated core sandwich beams and plates are compared in Fig. 9a and 9b, respectively. Recall that the sandwich plates have the longitudinal axis of the prismatic cores along the $X_{1}$ axis while the transverse core direction is aligned along the $X_{2}$ axis. However, the measured sandwich plate deflections are not intermediate to the sandwich beams with transverse and longitudinal core arrangements. Rather, the sandwich plates outperform the sandwich beams (in terms of $w_{b}$ ) with the longitudinal core orientation over the entire range of projectile velocities investigated here. This is presumably due to the enhanced stretch resistance of the face sheets in the clamped plate configuration. However, the enhanced stretching of the face-sheets results in tearing of the front face sheet at lower values of $I_{0}$ compared to the sandwich beams 
with the longitudinal core orientation as indicated in Fig. 9. Moreover, perforation of the sandwich plates occurs at $I_{0}=5.4 \mathrm{kNsm}^{-2}$ which is about $15 \%$ lower than that for sandwich beams with a longitudinal core orientation.

It is instructive to make a direct comparison of the deflections of the sandwich beams and plates with their monolithic counterparts. To address this, the ratio of measured permanent back face deflection at the centre of the sandwich plates to that of monolithic plates (of same mass) $w_{\text {sandwich }} / w_{\text {monolithic }}$ is plotted as a function of projectile momentum $I_{0}$ in Fig. 10. The data for sandwich and monolithic beams from Rubino et al. (2007a) are included in the figure. There is only a minor effect of core topology upon the mid-span deflection for the beams and plates. However, the sandwich plates and the beams with a longitudinal core orientation outperform the monolithic plates particularly at low impulse levels; in contrast, the sandwich beams with the transverse core orientation show negligible benefit over monolithic beams of equal mass.

\section{4.}

Finite element simulations

Finite element (FE) predictions for the monolithic and sandwich plates are presented in this section. All computations were performed using the explicit time integration version of the commercially available finite element code $\mathrm{ABAQUS}^{3}$ (version 6.5). We first briefly describe the details of these FE calculations.

Three-dimensional simulations of half models of the rectangular sandwich and monolithic plates were performed. Linear hexahedral elements (C3D8R in ABAQUS notation) were used to model the cylindrical foam projectiles of diameter $d=50.8 \mathrm{~mm}$ and length $l_{0}=51 \mathrm{~mm}$, with the elements swept about the cylindrical axis of the foam projectile. The projectiles had 51 elements in the axial direction and 25 elements along the radius. The sandwich and monolithic plates were modelled using four-noded shell elements (S4R in ABAQUS notation) for both the face-sheets and the core. Perfect bonding between the core and face sheets was assumed. All dimensions (face sheet thickness and core dimensions) were chosen to match the experimental values and an element size of $1.0 \mathrm{~mm}$ was employed for all calculations reported subsequently.

Symmetry boundary conditions were specified at mid-span $\left(X_{1}=0\right)$ and clamped boundary conditions were applied at $X_{1}=L$ and at $X_{2}= \pm b / 2$ by constraining all rotations and displacements to zero; see Fig. 2a. The loading was simulated by impact of a metal foam projectile: at the start of the simulation the projectile with an initial velocity $v_{o}$ was brought into contact with the front face sheet at the centre of

\footnotetext{
${ }^{3}$ Hibbit, Karlsson and Sorensen Inc.
} 
the rectangular plates. The "general contact" option in ABAQUS was employed to simulate contact between all adjacent surfaces. The general contact algorithm in ABAQUS enforced hard, no-friction contact interaction using a penalty algorithm.

\subsection{Material properties}

The AISI 304 stainless steel was treated as a J2-flow theory rate dependent solid of density $\rho_{f}=7900 \mathrm{kgm}^{-3}$, Young's modulus $E=210 \mathrm{GPa}$ and Poisson ratio $v=0.3$.

The uniaxial tensile true stress versus equivalent plastic strain curves at plastic strainrates $10^{-3} \mathrm{~s}^{-1} \leq \&^{p} \leq 10^{4} \mathrm{~s}^{-1}$ were tabulated in ABAQUS using the prescription described in Section 2.2 and employing the data of Fig. 5a.

The metal foam projectile was modelled as a compressible continuum using the constitutive model of Deshpande and Fleck (2000). An isotropic yield surface is defined by:

$$
\hat{\sigma}-Y=0 .
$$

where $Y$ is the current yield strength. The equivalent stress $\hat{\sigma}$ has the following form:

$$
\hat{\sigma}^{2} \equiv \frac{1}{1+(\alpha / 3)^{2}}\left[\sigma_{e}^{2}+\alpha^{2} \sigma_{m}^{2}\right]
$$

where the parameter $\alpha$ defines the shape of the yield surface, $\sigma_{m} \equiv \sigma_{k k} / 3$ is the mean stress and $\sigma_{e} \equiv \sqrt{3 s_{i j} s_{i j} / 2}$ is the von-Mises effective stress in terms of the deviatoric stress $s_{i j}$. The yield strength $Y$ is assumed to be given by an over-stress type relation

$$
Y=\mu \varepsilon^{d}+\sigma_{c},
$$

where $\mu$ is the foam viscosity, $\varepsilon^{b}$ is the work conjugate plastic strain rate to $\hat{\sigma}$ and $\sigma_{c}\left(\hat{\varepsilon}^{p}\right)$ is the uniaxial stress versus plastic strain relation at a negligible strain rate. In the simulations, the Alporas foam is ascribed a density of $\rho_{p}=313 \mathrm{kgm}^{-3}$, Young's modulus $E_{c}=1.0 \mathrm{GPa}$, an elastic Poisson's ratio $v=0.3$. Consistent with observations reported by Deshpande and Fleck (2000) we assumed $\alpha=3 / \sqrt{2}$ which implies that the "plastic Poisson's ratio" $v_{p}=0$. The yield strength $\sigma_{c}$ versus equivalent plastic strain $\hat{\varepsilon}^{p}$ history is calibrated using the compressive stress versus strain response presented in Fig. 5b. The assumed over-stress model smears the plastic shock wave in the foam projectile over a width (Radford et al., 2005):

$$
w=\frac{\mu \varepsilon_{D}}{\rho_{p} \Delta v},
$$

where $\varepsilon_{D}$ is the nominal densification strain and $\Delta v$ is the velocity jump across the shock. For the purposes of this discussion, $\Delta v$ is approximately equal to the projectile velocity $v_{o}$. The values of the viscosity $\mu$ were chosen so that $w=l_{0} / 10 \approx 5 \mathrm{~mm}$, which is approximately equal to that observed in the experiments 
of Radford et al. (2005). Large gradients in stress and strain occur over the shock width and thus the foam projectile was discretized by a mesh size of $1 \mathrm{~mm}$ in order to resolve these gradients accurately.

\subsection{Comparison of finite element predictions and measurements}

Representative FE calculations of the transient deflection of the centre point of the sandwich and monolithic plates are plotted in Fig. 11 for $I_{0}=3.2 \mathrm{kNsm}^{-2}$. The deflections of both the front and back face sheets of the sandwich plates are displayed. In all cases only small elastic vibrations occur after the maximum deflections have been attained, so that the peak deflection is close to the permanent deflection.

The predicted permanent deflections $w_{b}$ of the back face sheets of the sandwich plates and of the monolithic plates are included in Fig. 6a along with the corresponding measured values. The permanent deflections in the FE calculations are estimated by averaging the displacements over several cycles of elastic vibration (from trough to peak) immediately after the initial peak displacement. We conclude that at least for the lower values of $I_{0}$ where no tearing of the plates was observed, the FE simulations are able to predict the plate deflections with reasonable accuracy. Some of the discrepancies may be accounted for as follows:

(i) At the high values of $I_{0}$ the observed deflections of the monolithic plates are greater than the predictions as the plates start to pull out of the supports, see Fig. 7a.

(ii) The FE calculations overestimate $w_{b}$ for the Y-frame plates at intermediate values of $I_{0}$. We shall see subsequently that this is primarily due to the fact that the FE calculations underestimate the degree of core compression in the Y-frame plates.

The FE predictions for the final core compression $\varepsilon_{c}$ at the plate centre are compared with measurements in Fig. $6 \mathrm{~b}$ as a function of the applied momentum $I_{0}$. Good agreement between the measurements and predictions is achieved for the corrugated core, however the FE calculations under-predict the core compression in the Y-frame sandwich plates. The critical difference between the predictions and observations is that the FE calculations predict that the core compression remains constant at $\varepsilon_{c} \approx 0.4$ for $I_{0} \leq 3 \mathrm{kNsm}^{-2}$ while in the experiments core compression increases with increasing $I_{0}$ over the entire range of projectile velocities investigated here. We attribute this discrepancy to the fact that tearing between the Y-frame leg and the back face sheet was observed in all specimens but was not modelled in the FE calculations. It is also worth noting that the slight kink in the FE predictions of the deflections of the back face of the Y-frame plates (Fig. 6a) corresponds to the $I_{0}$ range over which the FE calculations predict that the core compression remains constant. 
Comparisons between the measured and predicted permanent deformation of the plates impacted at $I_{0} \approx 3 \mathrm{kNsm}^{-2}$ are included in Fig. 8. In both the experiments and the FE calculations the plates are sectioned along their mid-span $X_{1}=0$ to reveal the core deformation in the sandwich plates. In general good agreement is obtained although there are some discrepancies especially for the Y-frame sandwich plate as discussed above.

\subsection{Estimation of the onset of front face tearing}

The final plastic strain distribution within the $\mathrm{Y}$-frame and corrugated core sandwich plates was explored in the FE simulations for the choice $I_{0}=5.1 \mathrm{kNsm}^{-2}$. This impulse level was chosen as it gave front face tearing of both sandwich plates, but not complete perforation. The location and direction of the maximum principal strains in the sandwich plates are shown in Figs. 12a and $12 \mathrm{~b}$ for the Y-frame and corrugated core plates, respectively. For the Y-frame plate, the highest predicted strain occurs close to the joint between the Y-frame web and the front face sheet while for the corrugated core high values of final plastic strain occur both in the front face sheet and within the core itself. The plastic strain predictions of the FE calculations are generally consistent with the observation that failure always initiates in the front face sheet.

The FE predictions of the maximum principal plastic strains $\varepsilon_{\max }$ in the face sheets of the Y-frame and corrugated core plates are compared with those in the corresponding sandwich beams (longitudinal core orientation) in Fig. 13a over a range of values of $I_{0}$. The FE predictions for the beams are obtained from the parallel study by Rubino et al. (2007a). The FE calculations predict significantly higher values of $\varepsilon_{\max }$ in the plates than in the beams, especially for $I_{0} \geq 4 \mathrm{kNsm}^{-2}$; this is consistent with the observation that the plates fail at lower values of $I_{0}$ than the sandwich beams of longitudinal core orientation. Comparisons between the predicted values of $\varepsilon_{\max }$ for the sandwich and monolithic plates are included Fig. 13b. Recall that failure of the front face sheet was observed at lower values of $I_{0}$ in the Y-frame plate than the corrugated core plate. Also, no failure of the monolithic plate was observed even at the highest value of $I_{0}$ investigated here. Consistent with these observations, the FE calculations predict the lowest values of $\varepsilon_{\max }$ in the monolithic plates followed by the corrugated core plates, and the largest strains occur in the Y-frame plates.

\section{5.}


Metal foam projectiles have been used to impact clamped 304 stainless steel monolithic plates and sandwich plates with Y-frame or corrugated cores. The permanent deflections and level of core compression of the sandwich plates have been measured as a function of projectile momentum, and the measured responses are compared with finite element simulations. The finite element (FE) simulations capture the observed deformation modes to reasonable accuracy. Failure of the joints between the core and the face sheets is not included in the FE predictions, and this leads to some discrepancies especially for the Y-frame plates.

The sandwich plates outperform monolithic plates of equal mass at low values of the projectile momentum $I_{0}$. Moreover, comparisons with the corresponding Y-frame and corrugated core sandwich beams revealed that the plates deflect less than the beams. However, the benefits of sandwich construction reduce with increasing $I_{0}$ : (i) the ratio of the permanent deflections of the sandwich plates to monolithic plates increases with increasing $I_{0}$ and approaches unity for $I_{0} \approx 6 \mathrm{kNsm}^{-2}$; (ii) stress concentrations at the attachment points between the core and the face sheets result in failure of the sandwich plates at lower values of $I_{0}$ than for the monolithic plates. We conclude that sandwich construction has potential for significantly enhancing the dynamic performance of structures. However, sandwich construction must be used with caution in heavily loaded structures: the sandwich plates may fail at lower values of $I_{0}$ than for monolithic plates of equal areal mass.

\section{Acknowledgments}

This work was supported by the Netherlands Institute for Metal Research: project number MC1.03163, The Optimal Design of Y-core sandwich structures.

\section{References}

Côté, F., Deshpande V. S., Fleck N. A., Evans, A.G.,2006. The compressive and shear responses of corrugated and diamond lattice materials. International Journal of Solids and Structures 43, 6220-6242.

Deshpande V. S., Fleck N. A., 2000. Isotropic constitutive models for metallic foams. Journal of Mechanics and physics of Solids 48, 1253-1283.

Konter, A., and Broekhuijsen, J., and Vredeveldt, A., 2004. A quantitative assessment of the factors contributing to the accuracy of ship collision predictions with the finite element method. International Conference of Ship Collisions and Grounding, Tokyo, Japan.

Ludolphy, H., 2001. The Unsinkable Ship -Development of the Y-Shape Support Web. Proceedings of the $2^{\text {nd }}$ International Conference on Collision and Grounding of Ships, Copenhagen, Denmark.

McShane G.J., Radford D.D., Deshpande V.S. and Fleck N.A., 2006 The response of clamped sandwich plates with lattice cores subjected to shock loading. European Journal of Mechanics $25,215-229$. 
Naar, H., and Kujala, P., and Simonsen, B.C., and Ludolphy, H., 2002. Comparison of the Crashworthiness of Various Bottom and Side Structures. Marine Structures 15, 443-460.

Paik, J.K., 2003. Innovative Structural Designs of Tankers Against Ship Collisions and Grounding: A Recent State-of-the-Art Review. Marine Technology 40, 25-33.

Pedersen, C.B.W., and Deshpande, V. S., and Fleck, N.A., 2006. Compressive Response of the Y-Shaped Sandwich Core. European Journal of Mechanics-A/Solids 25, 125-141.

Radford, D.D., Fleck N.A., Deshpande V.S., 2005. The use of metal foam projectiles to simulate shock loading on a structure. International Journal of Impact Engineering 31, 11521171.

Radford, D.D., Fleck N.A., Deshpande V.S., 2006a. The response of clamped sandwich beams subjected to shock loading. International Journal of Impact Engineering 32, 968-987.

Radford D.D., McShane G.J., Deshpande V.S. and Fleck N.A., 2006b. The response of clamped sandwich plates with metallic foam cores to simulated blast loading. International Journal of Solids and Structures 43, 2243-2259.

Rathbun, H.J., Radford, D.D., Xue, Z., He, M.Y., Yang, J., Deshpande, V.S., Fleck, N.A., Hutchinson, J.W., Zok, F.W., Evans, A.G., 2006. Performance of metallic honeycomb-core sandwich beams under shock loading. International Journal of Solids and Structures 43, 17461763.

Rubino, V., Deshpande V.S., Fleck N.A., 2007a. The dynamic response of end-clamped sandwich beams with a Y-frame or corrugated core, submitted to International Journal of Impact Engineering.

Rubino, V., Deshpande V.S., Fleck N.A., 2007b. The collapse response of sandwich beams with a Y-frame core subjected to distributed and local loading, International Journal of Mechanical Sciences doi:10.1016/j.ijmecsci.2007.07.007.

Stout M. G., Follansbee P. S., 1986. Strain-rate sensitivity, strain hardening, and yield behaviour of 304L stainless steel. Trans. ASME: Journal of Engineering Materials Technology 108,344-353.

Tilbrook, M.T., Radford D.D., Deshpande V.S., Fleck N.A., 2007. Dynamic crushing of sandwich panels with prismatic lattice cores. International Journal of Solids and Structures 44, 6101-6123.

Wevers, L. J., Vredeveldt, A. W., 1999. Full Scale Ship Collision Experiments 1998, TNOreport 98-CMC-R1725, The Netherlands. 


\section{Figure captions}

Fig. 1: Sketch of the (a) Y-frame and (b) corrugated sandwich cores as used in ship hull construction. The core is sandwiched between the outer and inner hull of the ship.

Fig. 2: (a) Sketch of the clamped sandwich plate geometry and the loading arrangement. Geometry of half sandwich plate for (b) Y-frame and (c) corrugated sandwich cores investigated in this study. The cross-section is also shown for each topology. All dimensions are in $\mathrm{mm}$.

Fig. 3: The manufacturing sequence for the Y-frame sandwich plates. (a) The leg is inserted into the slots in the folded Y-frame web. (b) The leg is laser welded to the Yframe web. (c) The Y-frame core is spot welded on to the front face sheet of the sandwich plate. (d) An aluminium insert locates the core and face sheets and (e) the assembly is laser welded together.

Fig. 4: The clamping arrangement of the sandwich plates. (a) The steel inserts and epoxy filling of the core ends allow for high clamping pressures. (b) The steel frame for clamping of the plates. All dimensions are in $\mathrm{mm}$.

Fig. 5: (a) The measured quasi-static $\left(\mathscr{E}=10^{-3} \mathrm{~s}^{-1}\right)$ tensile stress versus strain response of the AISI 304 stainless steel and the estimated responses at three additional values of applied plastic strain rate, using the data of Stout and Follansbee (1986). (b) The quasi-static nominal compressive stress versus nominal strain response of the Alporas metal foam.

Fig. 6: Measured and FE predictions of the permanent (a) back face deflections $w_{b}$ and (b) core compression at the centre of the dynamically loaded sandwich plates, as a function of the foam projectile momentum $I_{0}$. The data for the monolithic plates are included. Symbols denote measurements while the continuous lines are the FE predictions. Front face sheet failure is denoted by encircling the salient data points of in (b).

Fig. 7: Photographs showing a view of the front face of the as-tested (a) monolithic (b) Y-frame and (c) corrugated core plates. $I_{0} \approx 5 \mathrm{kNsm}^{-2}$.

Fig. 8: Photographed sections of the mid-plane $X_{1}=0$ of the as-tested (a) monolithic (b) Y-frame and (c) corrugated core plates. $I_{0} \approx 3 \mathrm{kNsm}^{-2}$.

Fig. 9: Comparison of the measured permanent deflections $w_{b}$ at the centre of the back face of the (a) Y-frame and (b) corrugated core sandwich plates and beams, as a function of the foam projectile momentum $I_{0}$. The data are shown up to $I_{0}$ values at which face sheet failure is first observed. The sandwich beam data are from Rubino et al. (2007a) and are for the cases when the prismatic axis and transverse axis of the 
core are along the length of the beam; these orientations are labelled longitudinal and transverse, respectively.

Fig. 10: The ratio of measured back deflections of the sandwich plates to monolithic plate versus projectile momentum $I_{0}$. The data are shown up to $I_{0}$ values at which face sheet failure is first observed. The sandwich beam data are from Rubino et al. (2007a), with the prismatic and transverse axes of the core along the length of the beam; these two cases are labelled longitudinal and transverse, respectively.

Fig. 11: Finite element prediction of the temporal variation of the deflection of the centre of the monolithic and sandwich plates loaded with a metal projectile momentum of $I_{0}=3.2 \mathrm{kNsm}^{-2}$.

Fig. 12: Sketch representing the FE predictions for the location and direction of the maximum principal strain in the (a) Y-frame and (b) corrugated core sandwich plates. The beams were impacted by the foam projectile of $I_{0}=5.1 \mathrm{kNsm}^{-2}$.

Fig. 13: FE predictions of the maximum principal plastic strain in the front face sheet of the dynamically loaded sandwich plates. (a) Comparison of the Y-frame and corrugated core sandwich beams (longitudinal core orientation) and plates. (b) Comparison between the sandwich plates and monolithic plates. The FE predictions for the sandwich beams are taken from Rubino et al. (2007a).

\section{List of Tables}

Table 1: The measured dynamic responses of monolithic and sandwich plates. The symbol $\mathrm{X}$ denotes through-thickness tearing of the plates. 


\begin{tabular}{|c|c|c|c|c|c|c|}
\hline \multirow[b]{2}{*}{ SPECIMEN } & \multicolumn{3}{|c|}{ PROJECTILE } & \multicolumn{3}{|c|}{ RESPONSE } \\
\hline & $\begin{array}{l}I_{0}=\rho_{p} I_{0} V_{0} \\
\left(\mathrm{kNsm}^{-2}\right)\end{array}$ & $\begin{array}{c}\rho_{p} \\
\left(\mathrm{kgm}^{-3}\right)\end{array}$ & $\begin{array}{c}v_{0} \\
\left(\mathrm{~ms}^{-1}\right)\end{array}$ & $\begin{array}{c}W_{b} \\
(\mathrm{~mm})\end{array}$ & $\varepsilon_{c}$ & $\begin{array}{c}\text { Value of } \\
X_{2} \text { where } \\
\text { tearing } \\
\text { started } \\
(\mathrm{mm})\end{array}$ \\
\hline \multirow{6}{*}{ Monolithic } & 1.6 & 331 & 94 & 10.5 & - & - \\
\hline & 3.0 & 306 & 191 & 15 & - & - \\
\hline & 5.1 & 334 & 299 & 23 & - & - \\
\hline & 5.5 & 308 & 351 & 26 & - & - \\
\hline & 6.2 & 311 & 392 & 28.8 & - & - \\
\hline & 6.6 & 289 & 451 & 33 & - & - \\
\hline \multirow{4}{*}{$\begin{array}{l}\text { as-brazed } \\
\text { Y-core }\end{array}$} & 1.7 & 331 & 99 & 2 & 0.55 & - \\
\hline & 3.1 & 306 & 198 & 6 & 0.84 & 13 (weld) \\
\hline & 5.1 & 332 & 301 & 18 & 0.96 & 0 \\
\hline & 5.4 & 300 & 351 & $x$ & $x$ & 0 \\
\hline \multirow{4}{*}{ Corrugated } & 1.7 & 331 & 99 & 2.5 & 0.49 & - \\
\hline & 3.0 & 309 & 192 & 6 & 0.73 & - \\
\hline & 5.0 & 332 & 297 & 16.5 & 0.96 & 10 \\
\hline & 5.4 & 300 & 351 & $x$ & $x$ & 26 (weld) \\
\hline
\end{tabular}

Table 2: The measured dynamic response of monolithic and sandwich plates. The symbol $X$ denotes through-thickness tearing of the plates. Tearing of the specimens started at $X_{1}=0$ and at a value of $X_{2}$ given in the last column. See Fig. 2 for a definition of the coordinate system. 
(a)

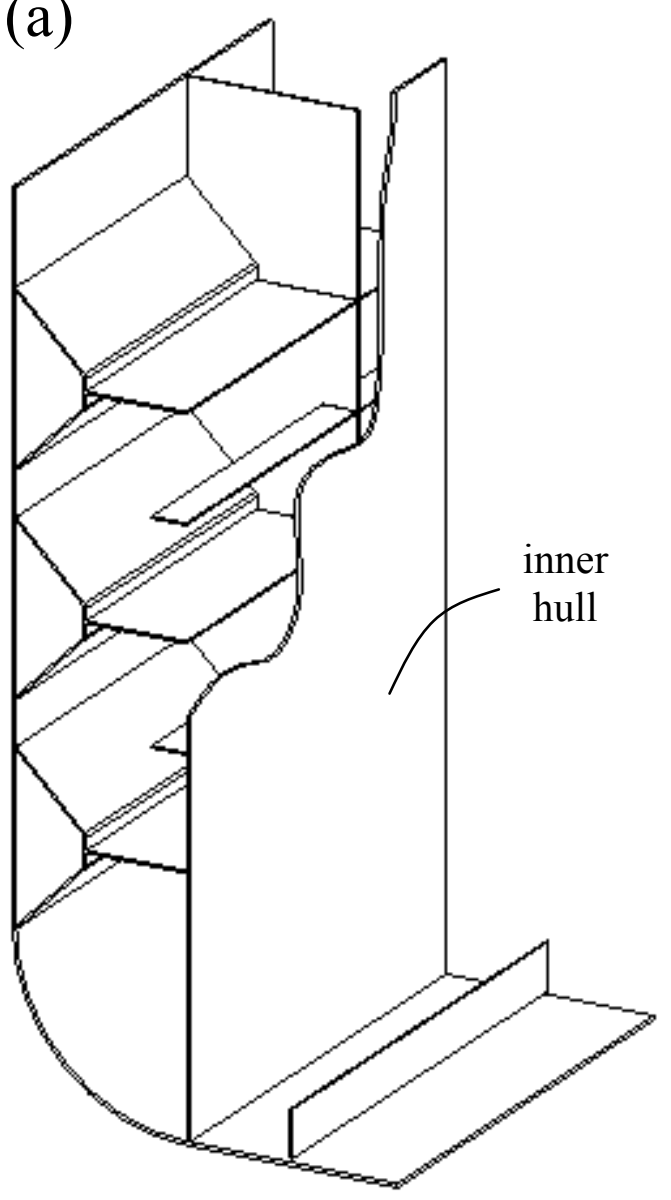

(b)

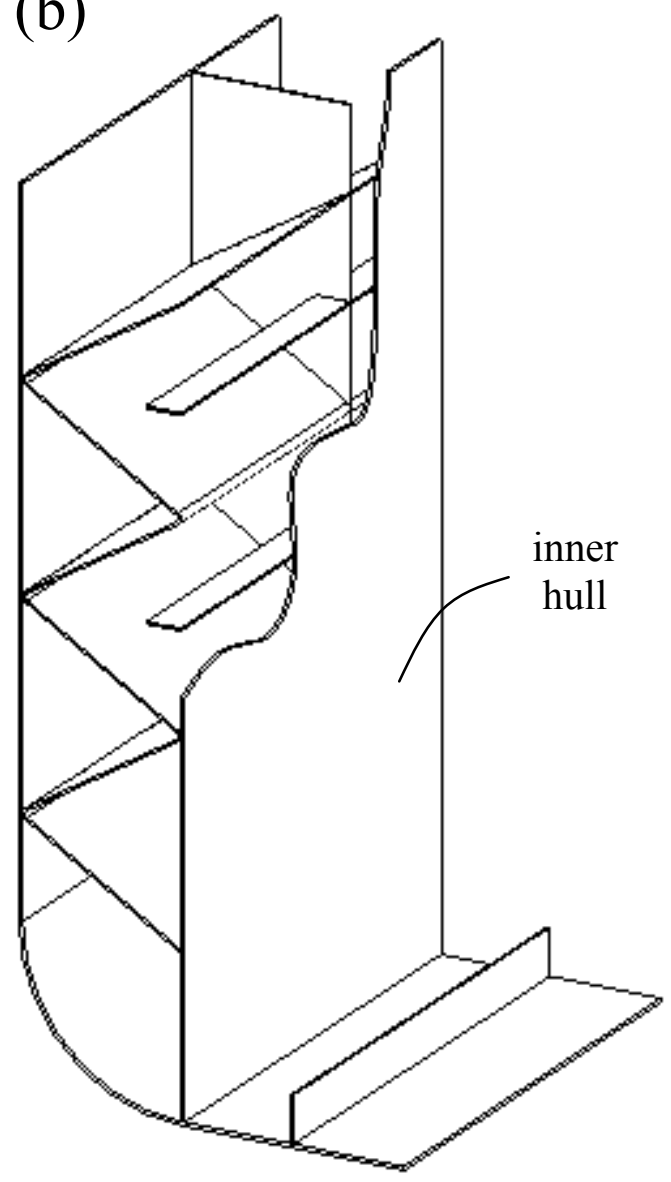

Fig. 1: Sketch of the (a) Y-frame and (b) corrugated sandwich cores as used in ship hull construction. The core is sandwiched between the inner and outer hulls of the ship. 
(a)

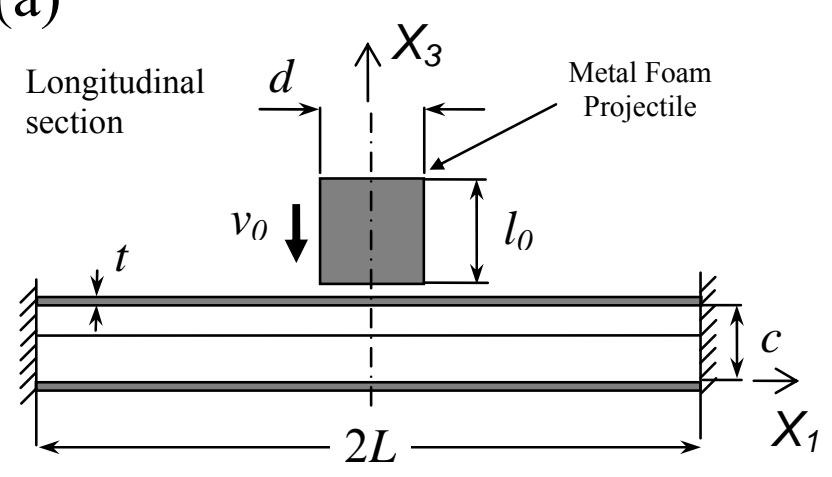

\section{Cross section}

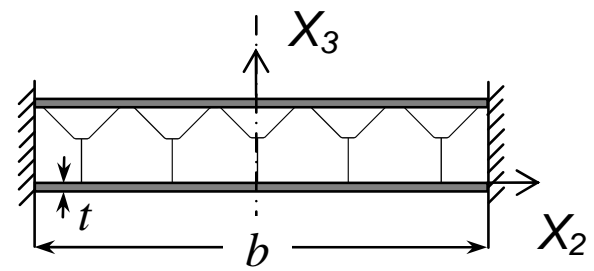

(b)

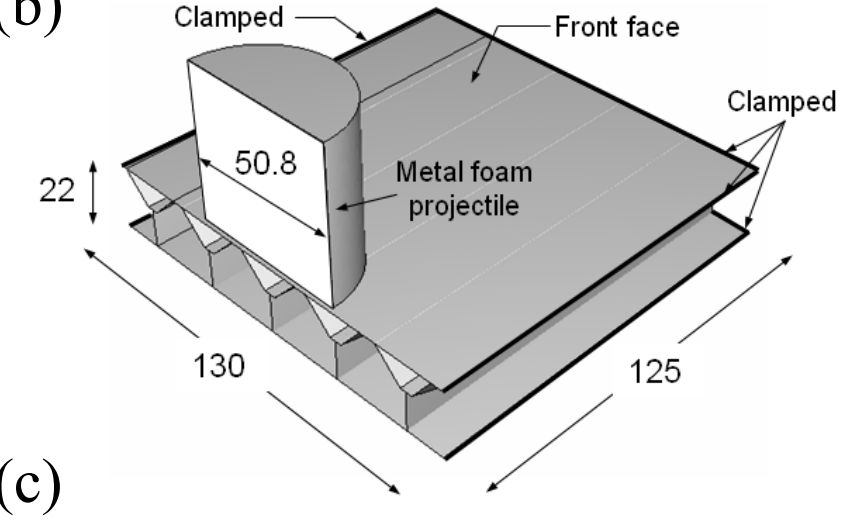

(c)

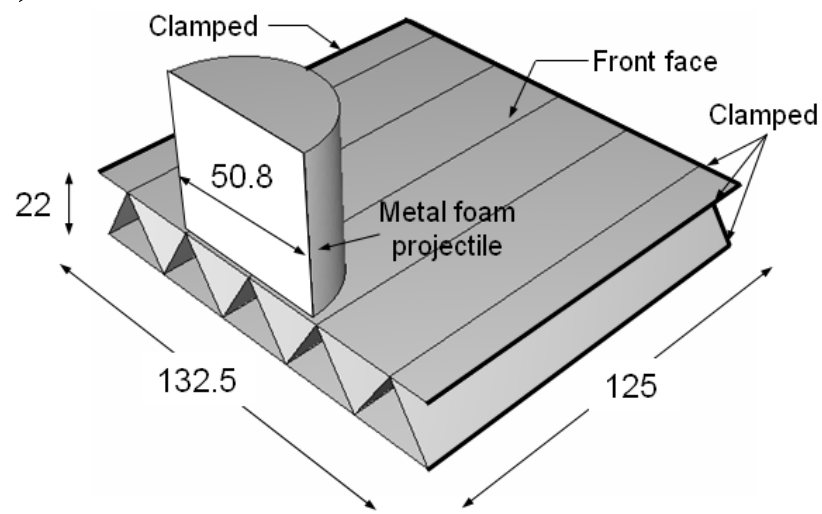

Dimensions in $\mathrm{mm}$
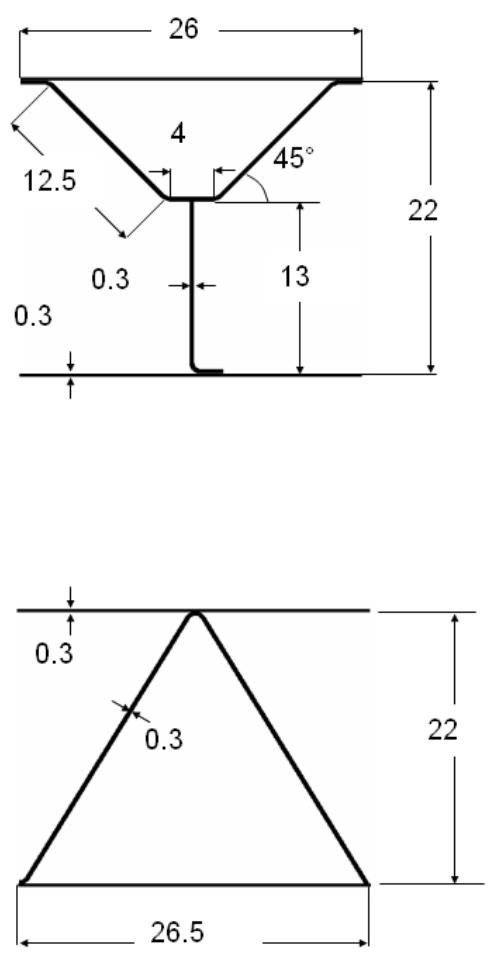

Fig. 2: (a) Sketch of the clamped sandwich plate geometry and the loading arrangement. Geometry of half sandwich plate for (b) Y-frame and (c) corrugated sandwich cores investigated in this study. The cross-section is also shown for each topology. All dimensions are in $\mathrm{mm}$. 
(a)
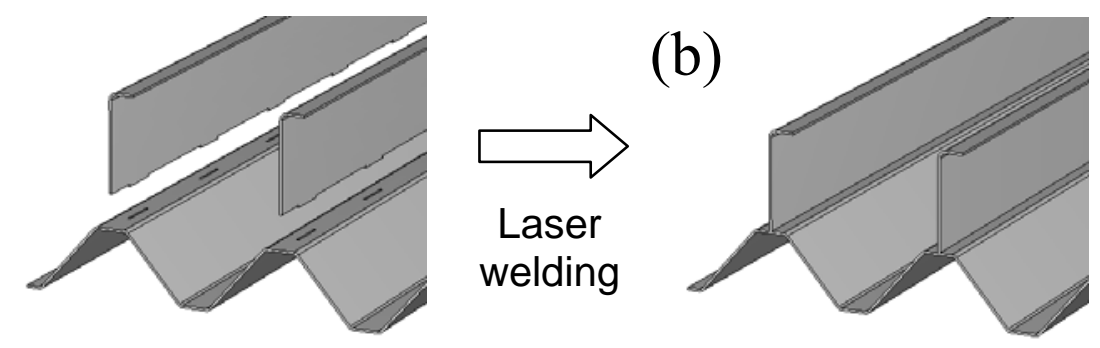

(c)

(d)

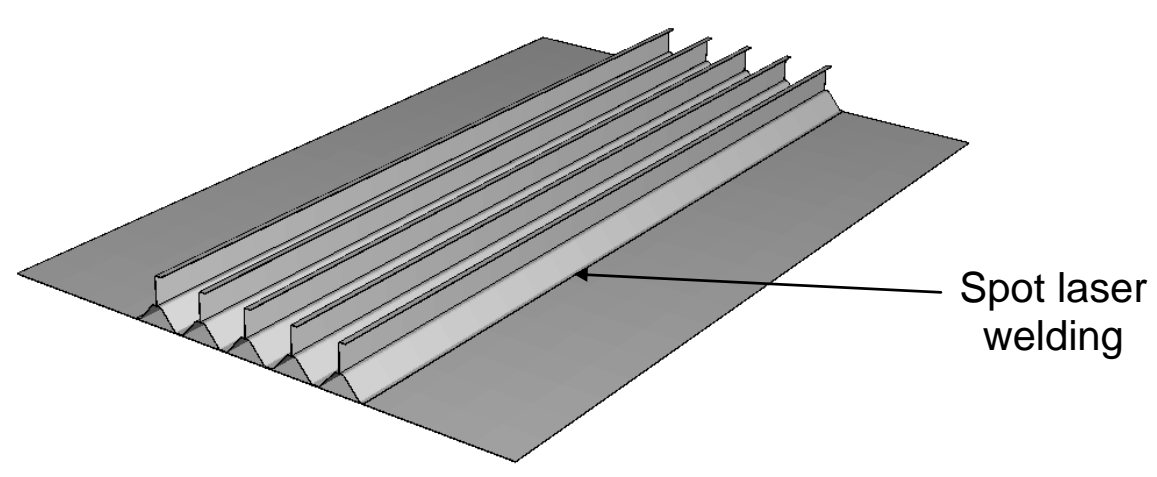

d)

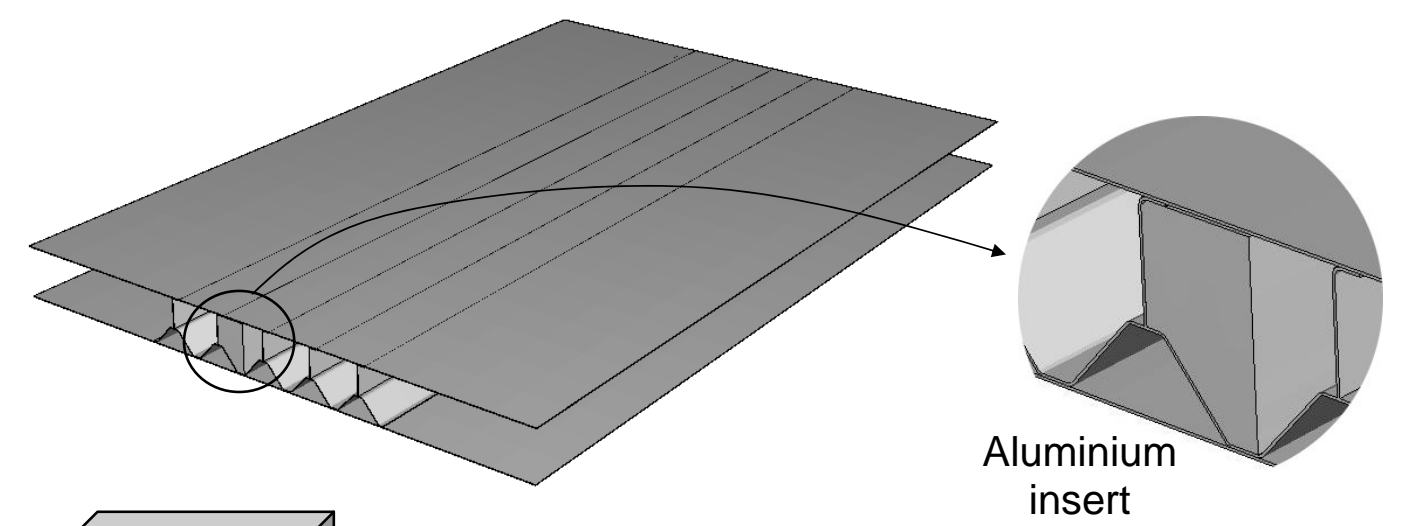

(e)

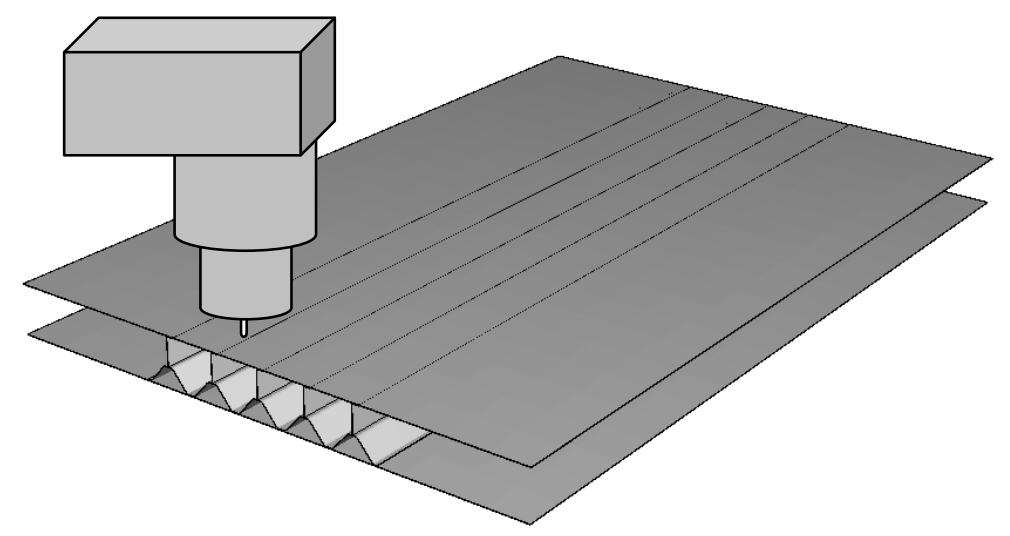

insert

Fig. 3: Manufacturing process for the laser welding of Y-frame sandwich plates. (a) The leg is inserted into the correspondent slots of the main frame and (b) is next manually welded on it along the $375 \mathrm{~mm}$ long side. (c) The Y-frame core is laser spot welded on the front face sheet with spots every $10 \mathrm{~mm}$, (d) the back face sheet is added with laser spot welding. An aluminium tool (shown in the inset) is here used to push together the two components to be spot welded. (e) A robot laser welds along the pre-spot welded lines. 


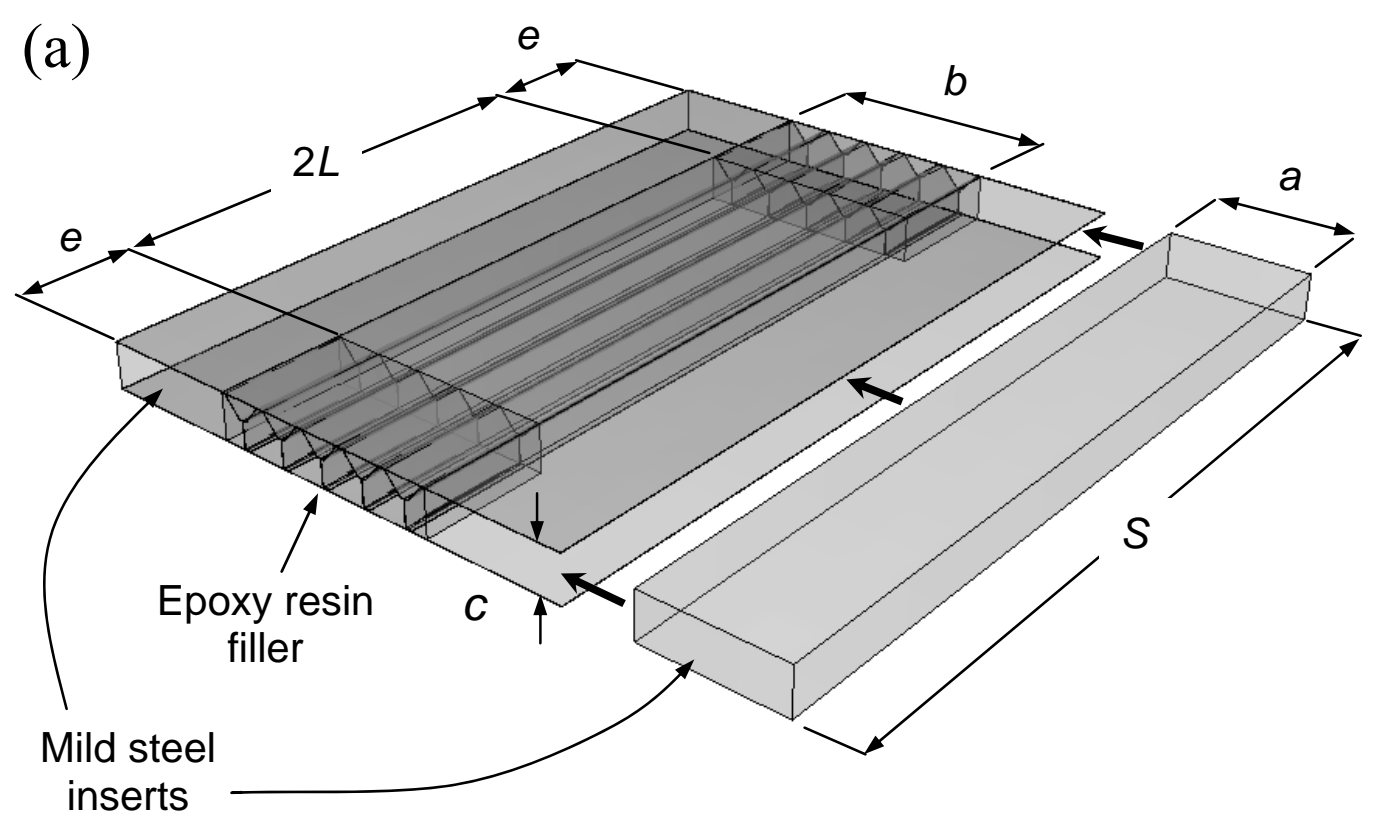

(b)

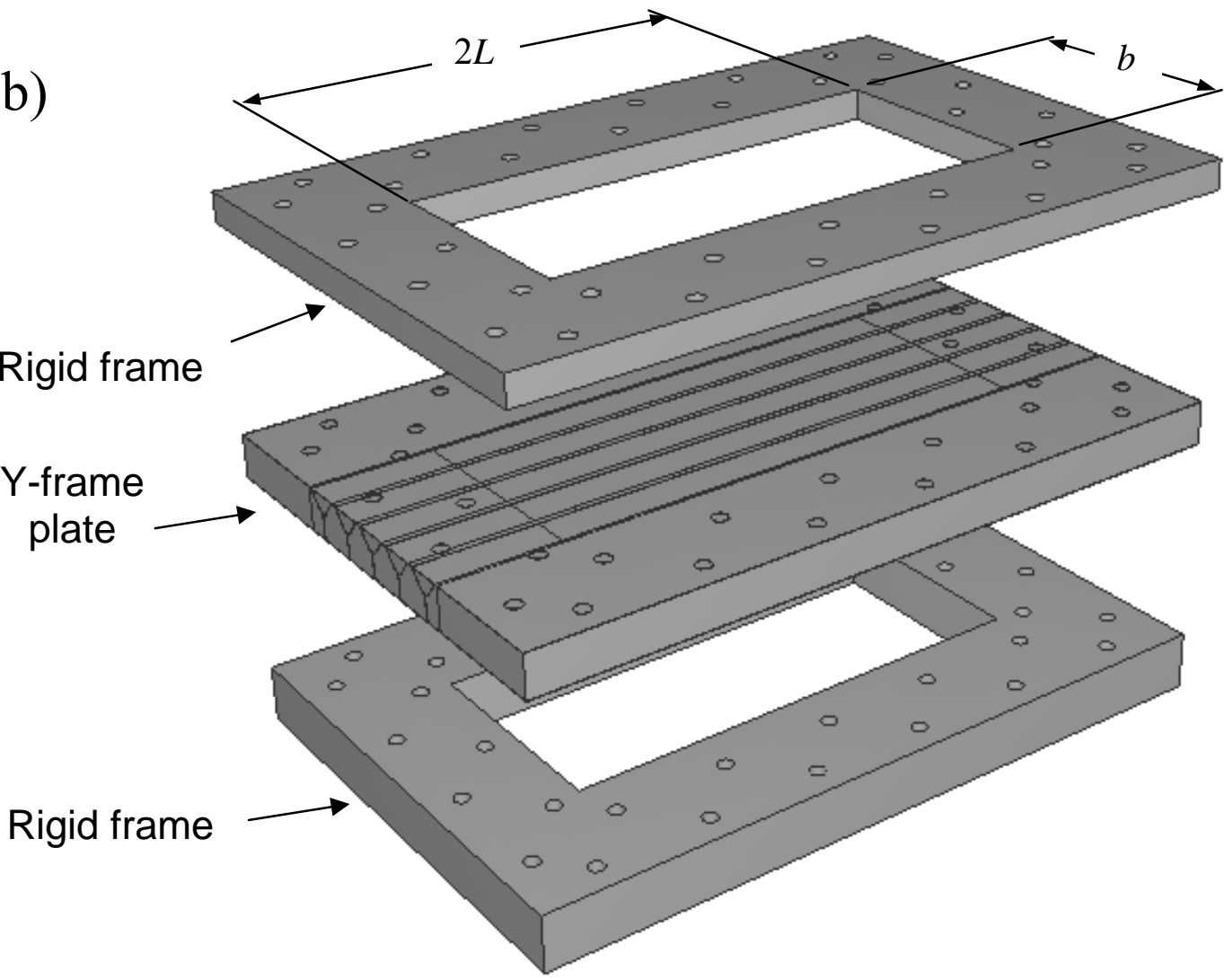

Fig. 4: Sketches of the manufacturing technique used to clamp the rectangular plates. (a) Mild steel inserts are glued between the face sheets of the long sides. A two part epoxy resin is poured in the two extremities of the plates. (b) The plate is bolted between two rigid rectangular frames. 
(a)

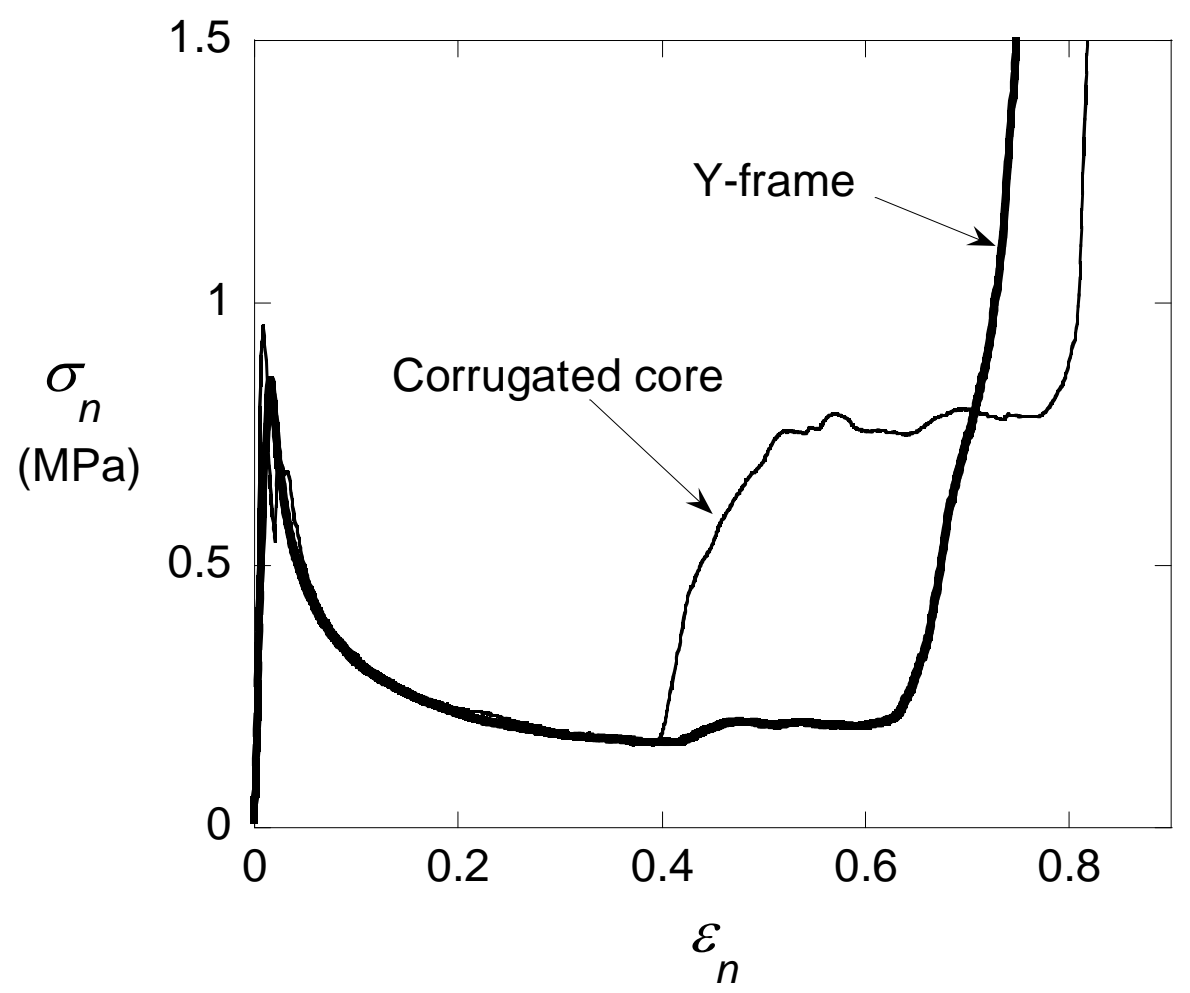

(b)

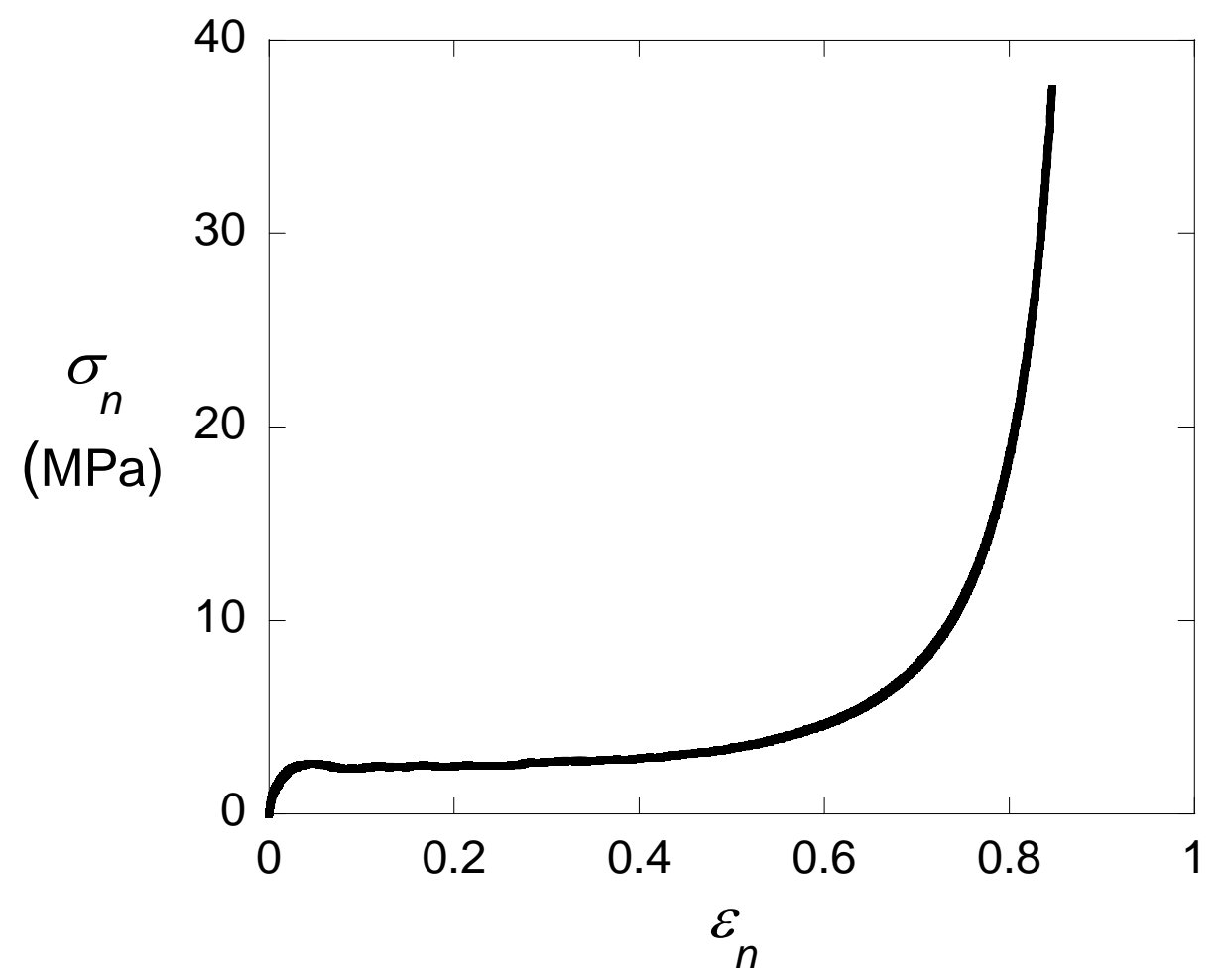

Fig. 5: (a) Measured quasi-static compressive stress versus strain responses of Yframe and corrugated sandwich cores. (b) The quasi-static nominal compressive stress versus nominal strain response of the Alporas metal foam. 
(a)

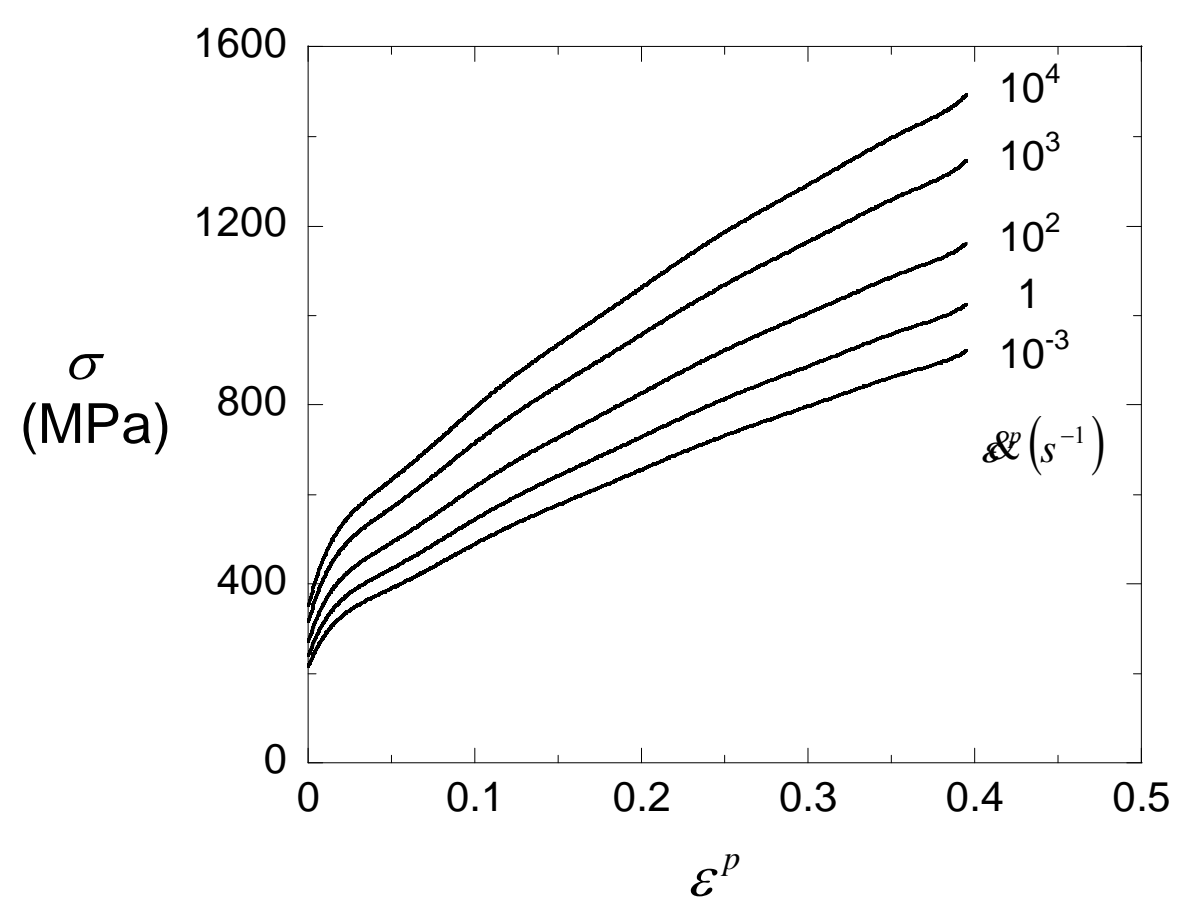

(b)

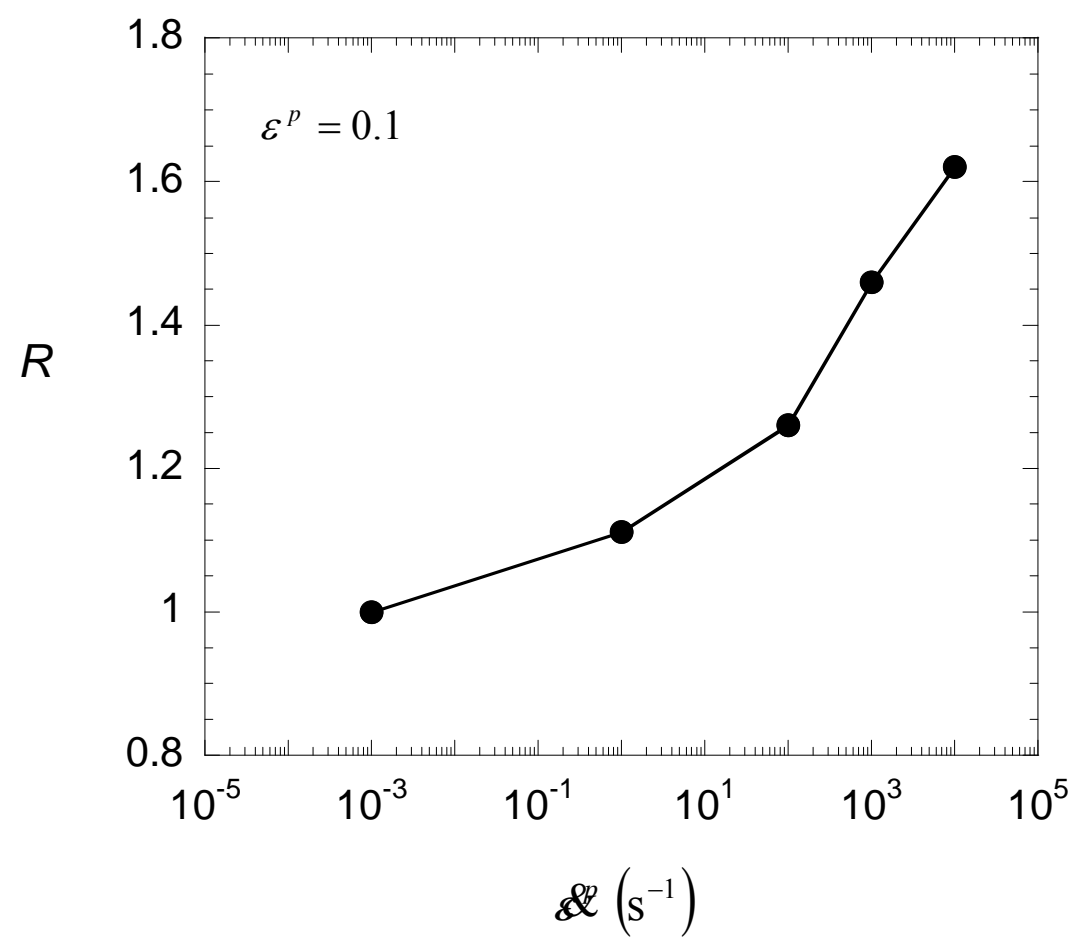

Fig. 6: (a) The measured quasi-static $\left(\& \mathbb{Q}=10^{-3} \mathrm{~s}^{-1}\right)$ tensile stress versus strain response of the AISI 304 stainless steel and the estimated high strain rate response at three additional values of strain rate, using the data of Stout and Follansbee (1986). (b) The dynamic strength enhancement ratio $R$ as a function of plastic strain-rate $\&$ for the AISI 304 stainless steel at a plastic strain $\varepsilon^{p}=0.1$. 
- Monolithic

- Y-frame

(a)

Corrugated

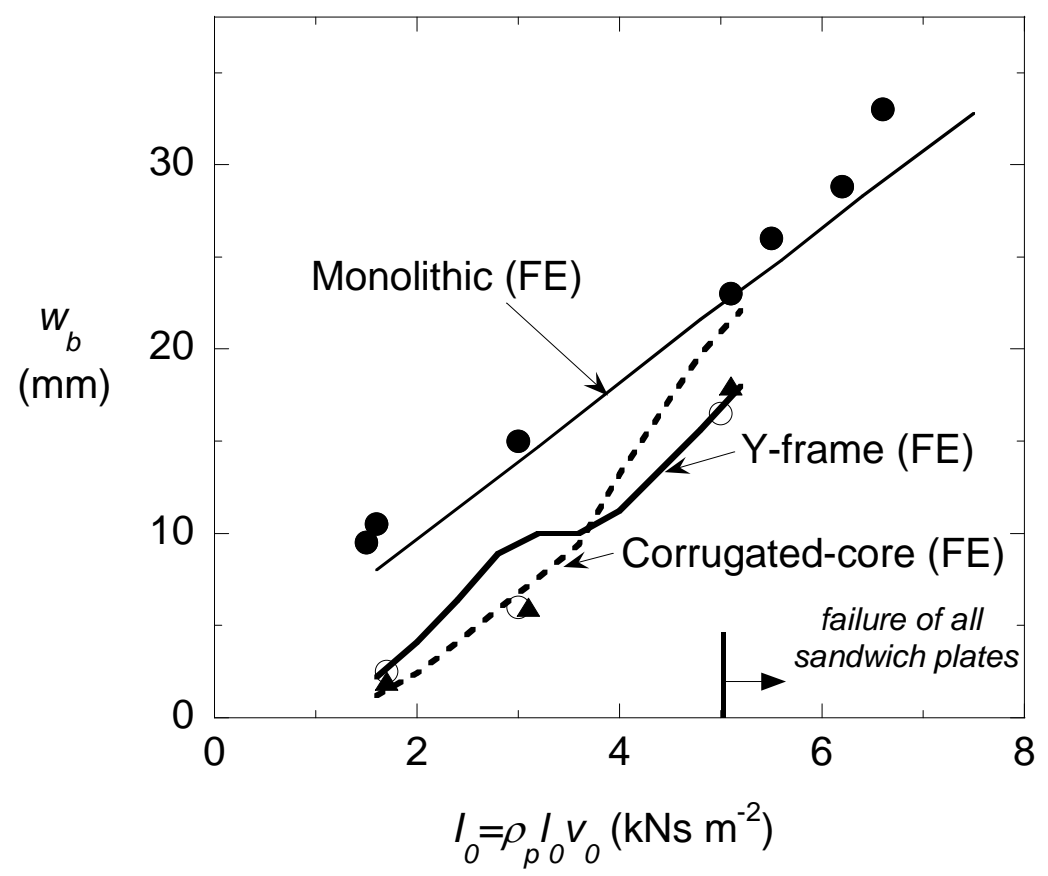

(b)

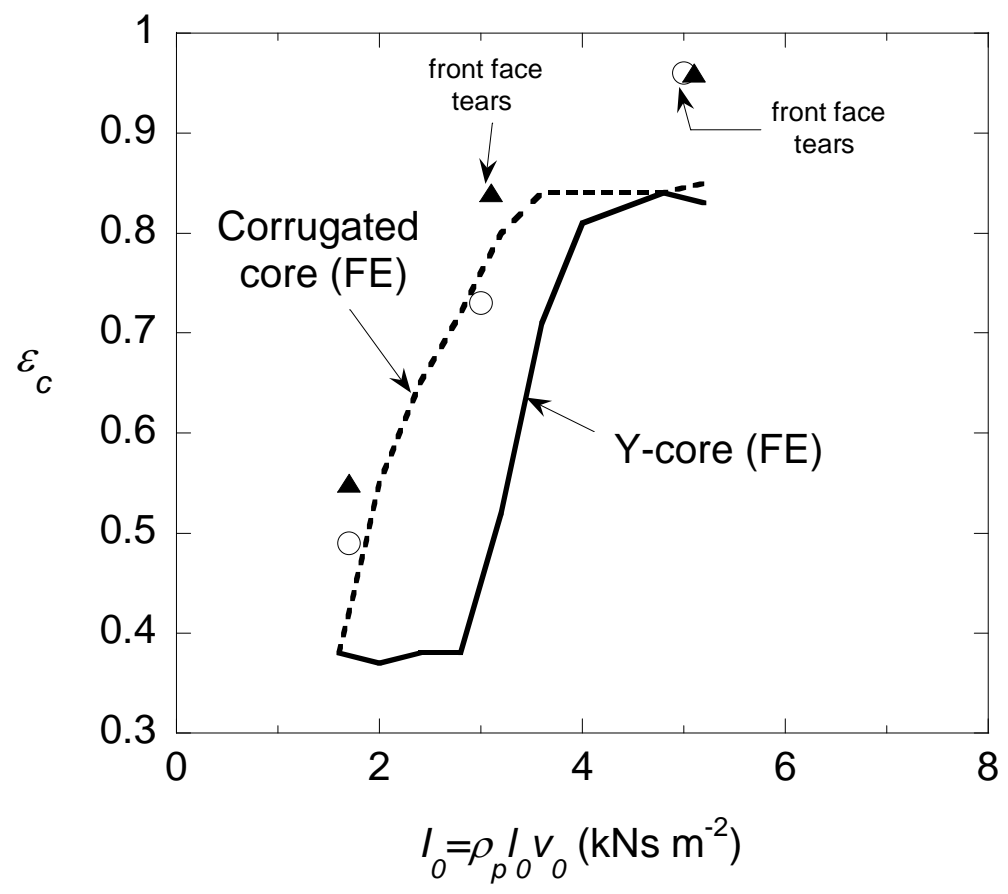

Fig. 7: Comparison of the measured and predicted permanent (a) rear-face deflections and (b) core compression at mid-span of the dynamically loaded monolithic and sandwich plates, as a function of the foam projectile momentum $I_{0}$. Symbols denote measurements and the continuous lines denote FE predictions. 
(a)

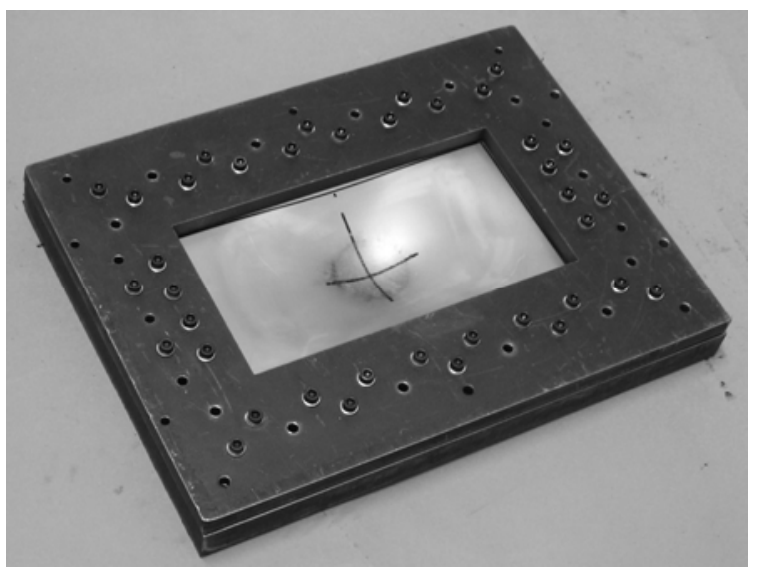

(b)

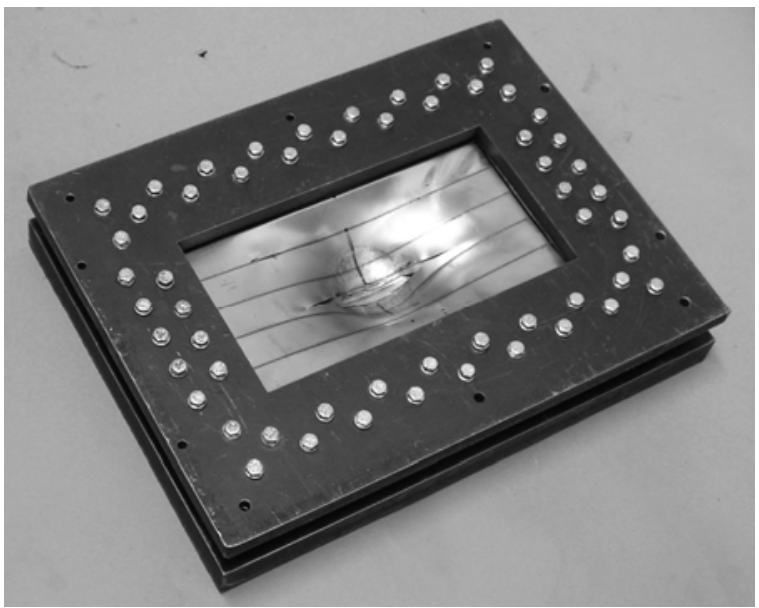

(c)

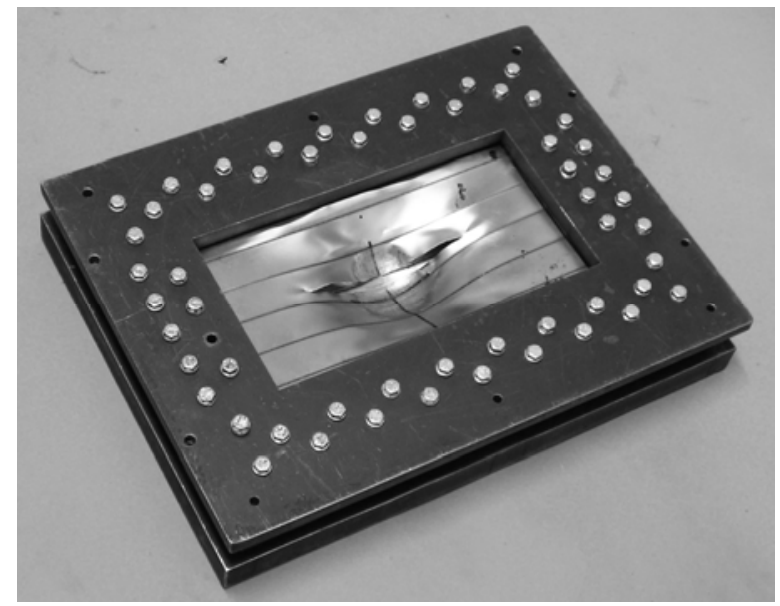

Fig. 8: Photographs of the as-tested (a) monolithic, (b) Y-frame and (c) corrugated core sandwich plates at approximately the same value of the foam projectile initial momentum $I_{0} \approx 5 \mathrm{kNsm}^{-2}$. Front face tearing is observed in both the sandwich plates. 


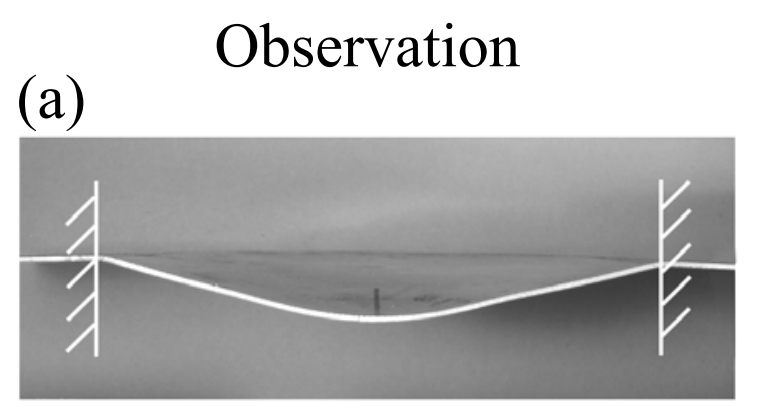

\section{Prediction}

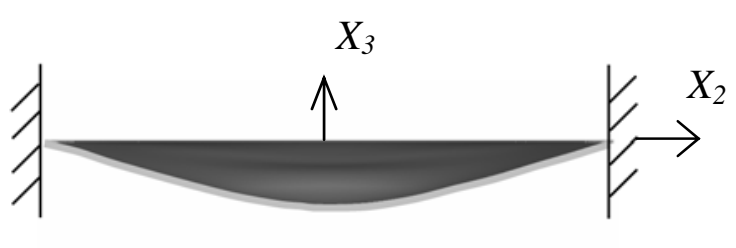

(b)
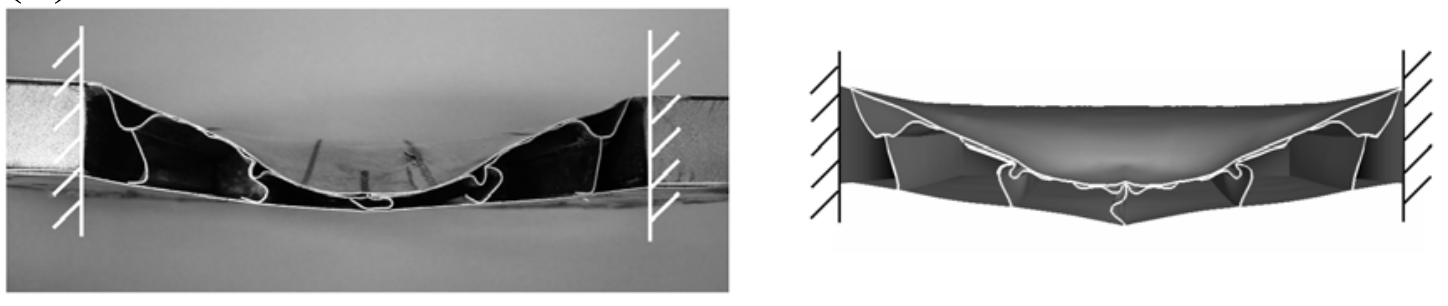

(c)
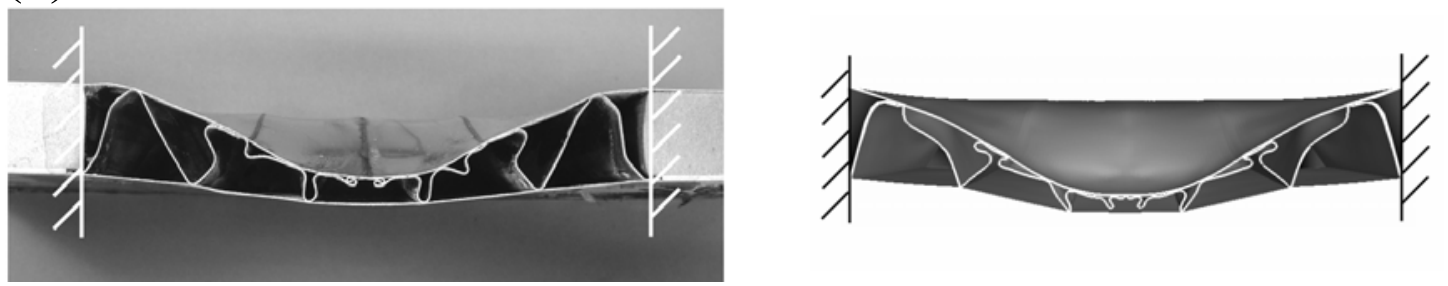

Fig. 9: Comparison between the observed and FE predicted deformed profiles of the plates sectioned at mid-span. (a) Monolithic plates, (b) Y-frame and (c) corrugated core sandwich plates impacted by the foam projectile at $I_{0} \approx 3 \mathrm{kNsm}^{-2}$. 
(a)

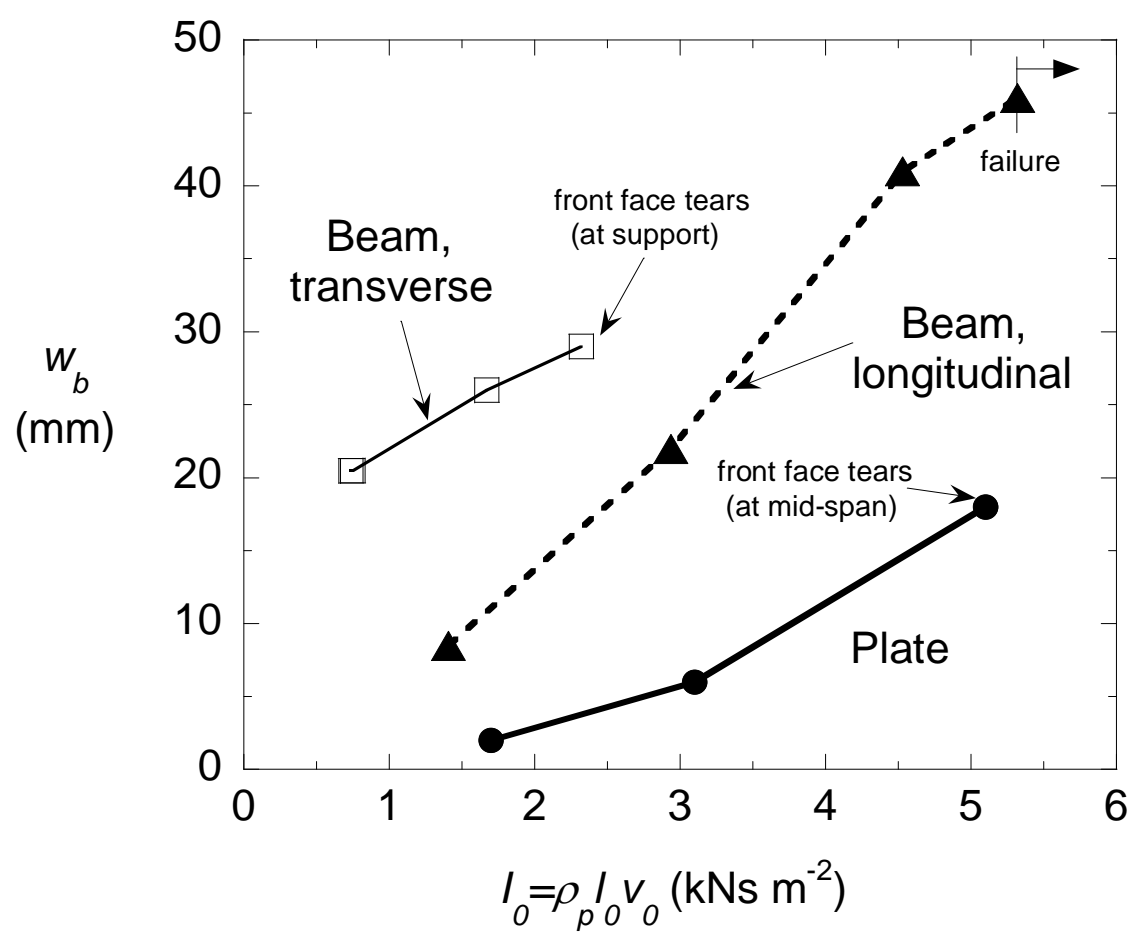

(b)

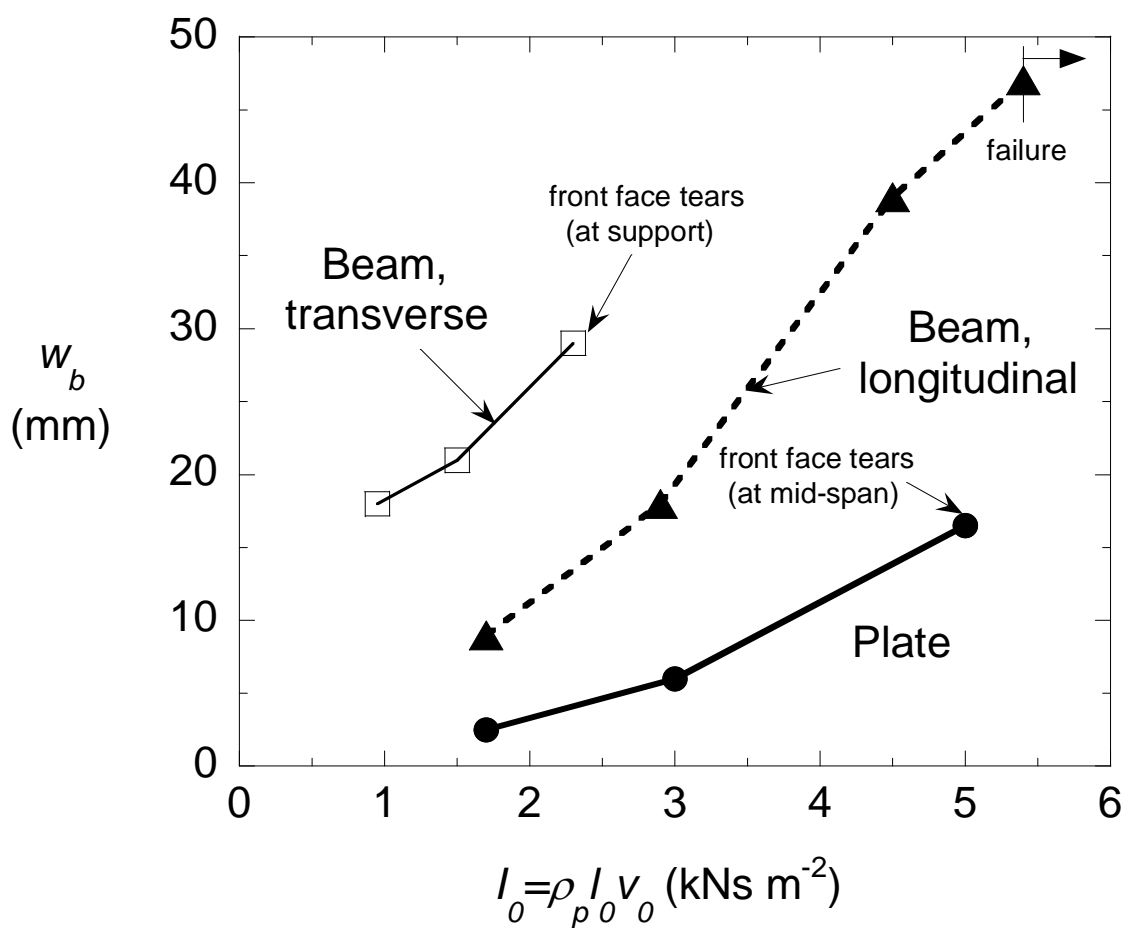

Fig. 10: Comparison of the measured permanent rear-face deflections at mid-span of the dynamically loaded (a) Y-frame and (b) corrugated core sandwich structures, as a function of the foam projectile momentum $I_{0}$. The response of the sandwich plates (Fig. 7a) is compared to that of sandwich beams with either longitudinal or transverse cores (see Rubino et al., 2007c). 


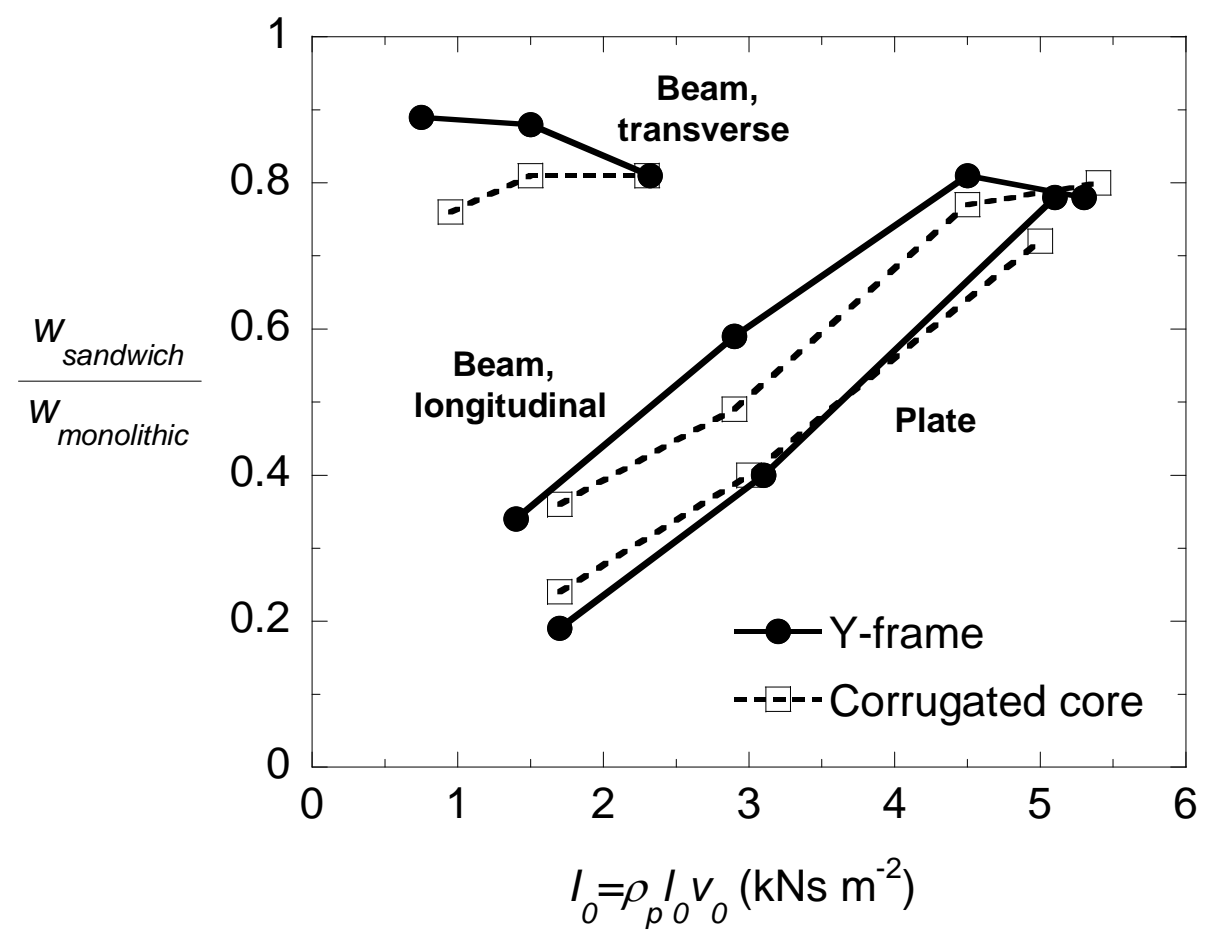

Fig. 11: Comparison of the measured normalized permanent rear-face deflections at mid-span of the dynamically loaded Y-frame and corrugated core sandwich structures, as a function of the foam projectile momentum $I_{0}$. The rear-face deflection of the sandwich structure is normalized with the rear-face deflection of the corresponding monolithic structure of the same mass. 


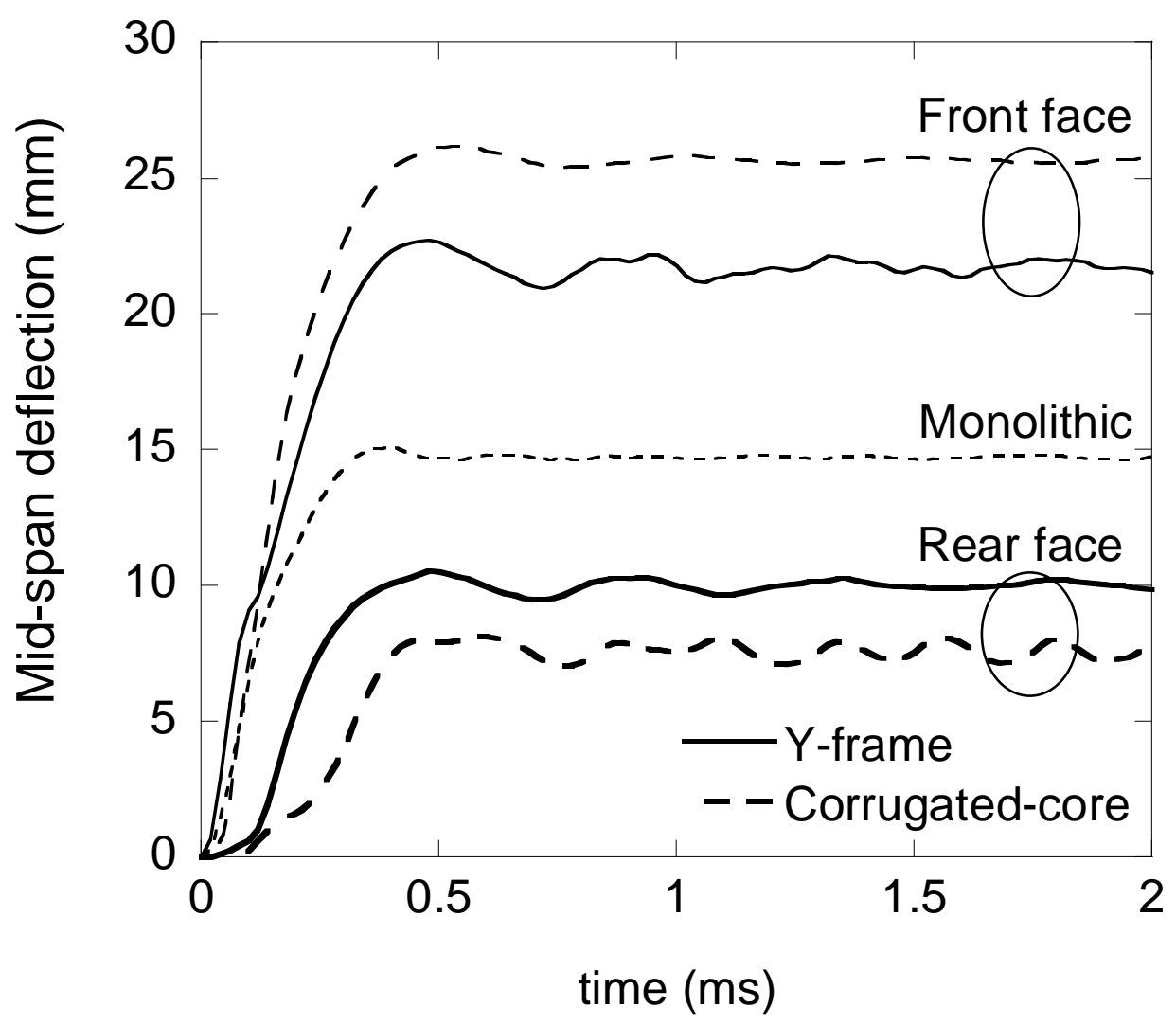

Fig. 12: Finite element prediction of the mid-span deflection versus time histories for monolithic and sandwich plates with $\mathrm{Y}$-frame or corrugated core loaded with a metal projectile momentum of $I_{0}=3.2 \mathrm{kNsm}^{-2}$. 
(a)
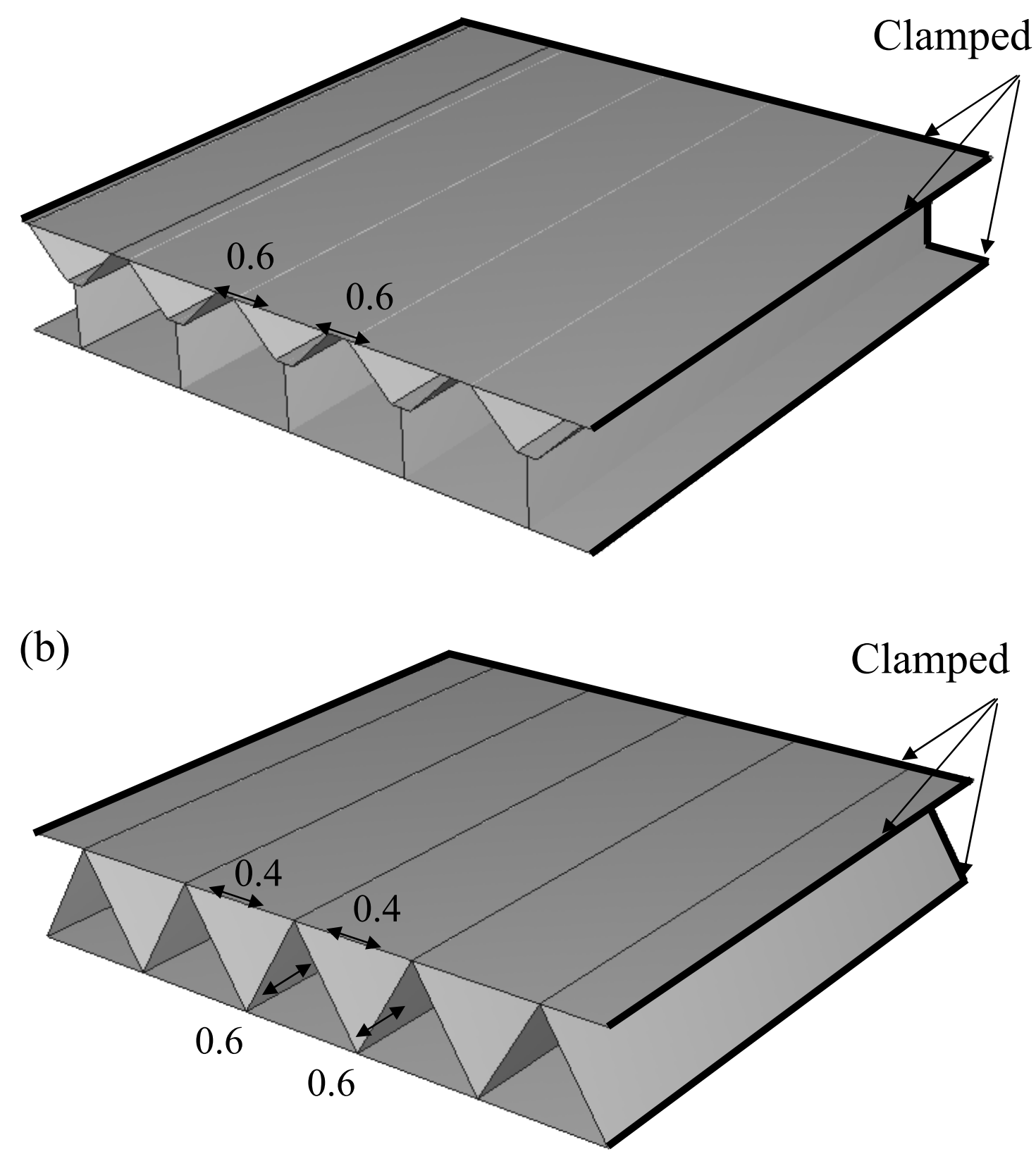

Fig. 13: Sketch showing the FE predictions for the location and direction of the maximum principal strains of the (a) Y-frame and (b) corrugated core sandwich plates. The clamped plates were impacted with the foam projectile momentum of $I_{0}=5.1 \mathrm{kNsm}^{-2}$. 
(a)

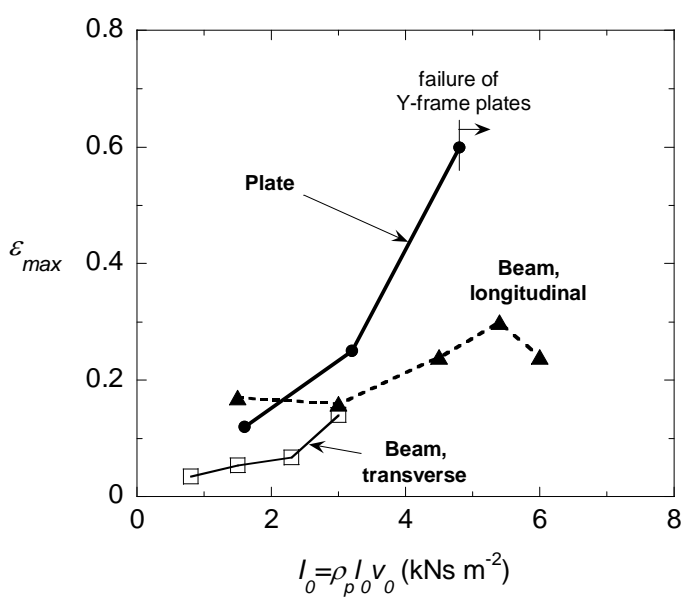

(b)

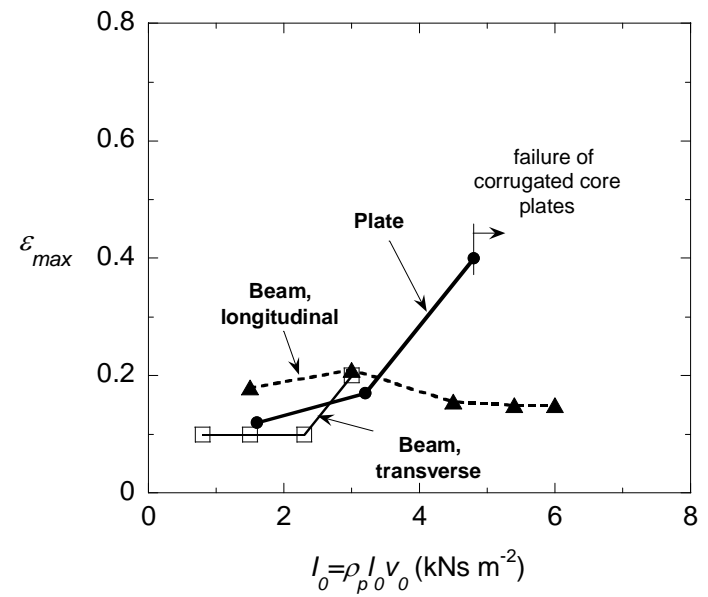

(c)

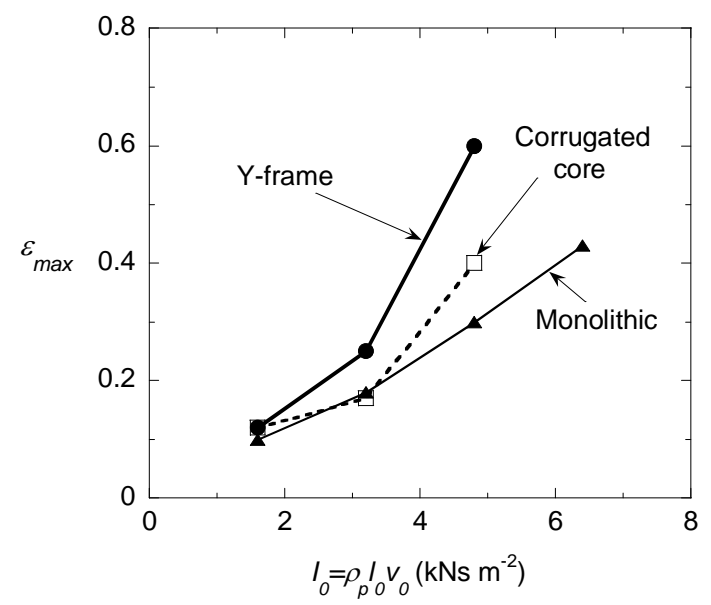

Fig. 14: Maximum principal plastic strain of the front face at mid-span of the dynamically loaded (a) Y-frame and (b) corrugated core sandwich structures, as a function of the foam projectile momentum $I_{0}$. The response of the plates is compared to beams with either longitudinal or transverse cores, see Rubino et al. (2007c). (c) Comparison of the maximum principal plastic strain in the front face of the sandwich plates and monolithic plates. 